\title{
KINEMATIC PAIRS: NOVEL KINDS AND SCIENTIFIC CLASSIFICATION
}

\author{
Stephen P. Radzevich \\ EATON Corp., Detroit, USA \\ radzevich@usa.com
}

\begin{abstract}
This paper deals with kinematic pairs in the most general sense, and, in particular, with those used in gear design. The paper begins with a brief overview of the earlier performed research in the field. Different kinds of kinematic pairs are distinguished, namely, point-contact kinematic pairs, line-contact kinematic pairs, and surface-to-surface-contact kinematic pairs. Contact geometry of functional surfaces in kinematic pairs is involved into the analysis aiming more in detail analysis of design, and the development of a scientific classification of kinematic pairs. A scientific classification of all possible kinds of kinematic pairs is developed. All known designs of kinematic pairs are covered by the classification. Moreover, kinematic pairs of all not known yet designs are also covered by the classification. This means that the proposed classification of kinematic pairs possesses predictive properties.

Key words: Kinematic pair, Functional surface, Contact geometry, Indicatrix of conformity, Highconformal contact, Scientific classification.
\end{abstract}

\section{КИНЕМАТИЧЕСКИЕ ПАРЫ: НОВЫЕ ВИДЫ И НАУЧНАЯ КЛАССИФИКАЦИЯ}

\author{
Степан Павлович Радзевич \\ EATON Corp., Detroit, USA \\ radzevich@usa.com
}

\begin{abstract}
Аннотация. $\quad$ этой статье рассматриваются кинематические пары в самом общем смысле и, в частности, те, которые используются в конструкции зубчатых колес. Статья начинается с краткого обзора ранее проведенных исследований в данной области. Различают кинематические пары с точечным контактом, кинематические пары $c$ линейным контактом и кинематические пары с контактом по поверхности. Контактная геометрия функциональных поверхностей в кинематических парах задействована в анализе с иелью более детального анализа конструкции и разработки научной классификации кинематических пар. Разработана научная классификачия всех возможных видов кинематических пар. Классификация охватывает все известные конструкции кинематических пар. Кроме того, под классификацию попадают кинематические пары всех еще не известных конструкиий. Это означает, что предложенная классификация кинематических пар обладает прогностическими свойствами.
\end{abstract}

Ключевые слова. кинематическая пара, функциональная поверхность, геометрия контакта, индикатриса конформности, высококонформный контакт, научная классификация.

Introduction. The investigation of machines began in the ancient times. A simple machine uses a single applied force to do work against a single load force. The concept of simple machine was 
helpful at the beginning of the human activity in the area of machine design. The idea of a simple machine originated with the Greek philosopher Archimedes around the $3^{\text {rd }}$ century $\mathrm{BC}$ who investigated the so-called simple machines. Usually, the term refers to the six classical simple machines that were defined by Renaissance scientists [16]:

- Wheel \& axle - makes work easier by moving objects across distances. The wheel (or round end) turns with the axle (or cylindrical post) causing movement

- Inclined plane - a flat surface (or plane) that is slanted, or inclined, so it can help move objects across distances

- Wedge - instead of using the smooth side of the inclined plane to make work easier, the pointed edges to do other kinds of work can be used

- Lever - any tool that pries something loose is a lever. Levers can also lift objects. A lever is an arm that "pivots" (or turns) against a fulcrum (the point or support on which a lever pivots)

- Pulley - instead of an axle, a wheel could also rotate a rope, cord, or belt. This variation of the wheel and axle is the pulley. In a pulley, a cord wraps around a wheel. As the wheel rotates, the cord moves in either direction

- Screw - when an inclined plane is wrapped around a cylinder, its sharp edge becomes a screw. A screw is actually just another kind of inclined plane.

The identification of simple machines arises from a desire for a systematic method to invent new machines. Therefore, an important concern is how simple machines are combined to make more complex machines. One approach is to attach simple machines in series to obtain compound machines.

A more successful strategy was identified by Franz Reuleaux. Reuleaux realized that a lever, pulley, and wheel and axle, are in essence the same device: a body rotating about a hinge. Similarly, an inclined plane, wedge, and screw are a block sliding on a flat surface.

This realization shows that it is the joints, or the connections that provide movement, that are the primary elements of a machine. Starting with four types of joints, the revolute joint, sliding joint, cam joint, and gear joint, it is possible to understand a machine as an assembly of solid parts that connect these joints.

Below in this paper, an abridged discussion of the results of the research that can be traced back to 1978-1981, and in detail disclosed in [16], is outlined.

1. Kinematic Pairs: Basics. Kinematic pair is one of the fundamental concepts in the mechanisms and machine theory. Kinematic pairs can be found out in the design of every machine, and of every mechanism. Examples of kinematic pairs of different kinds in gearing [(a) point-contact kinematic pair in the design of skew-axes gearing, (b) line-contact kinematic pair in the design of helical gearing, and (c) surface-to-surface-contact kinematic pair in the design of gear coupling] are illustrated in Fig. 1. Similar examples of kinematic pairs used in design of mechanisms of other kinds can also be provided. Taking into account the importance of the kinematic pairs to the science and practice of machines and mechanisms, properties of kinematic pairs of all kinds are required to be comprehensively investigated. 


\section{Bulletin of Science and Technical Development}

WwW.vntr.ru

№ 161, 2021

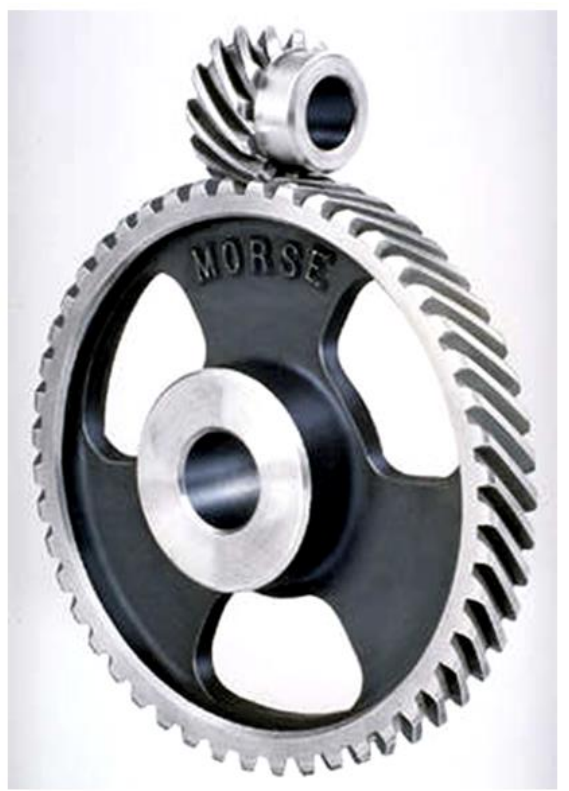

(a)

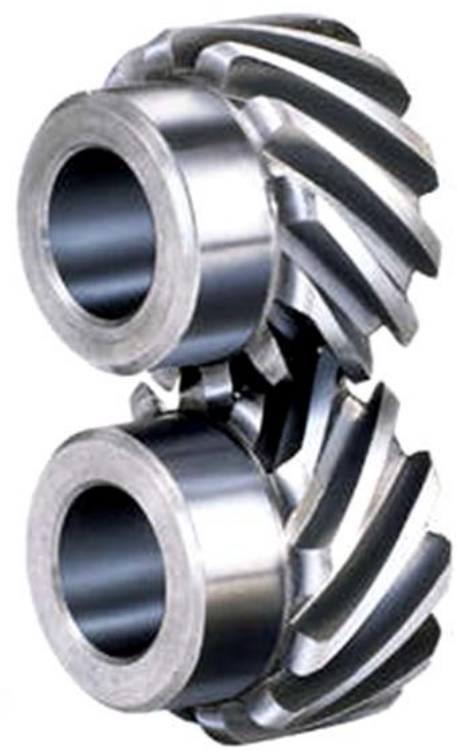

(b)

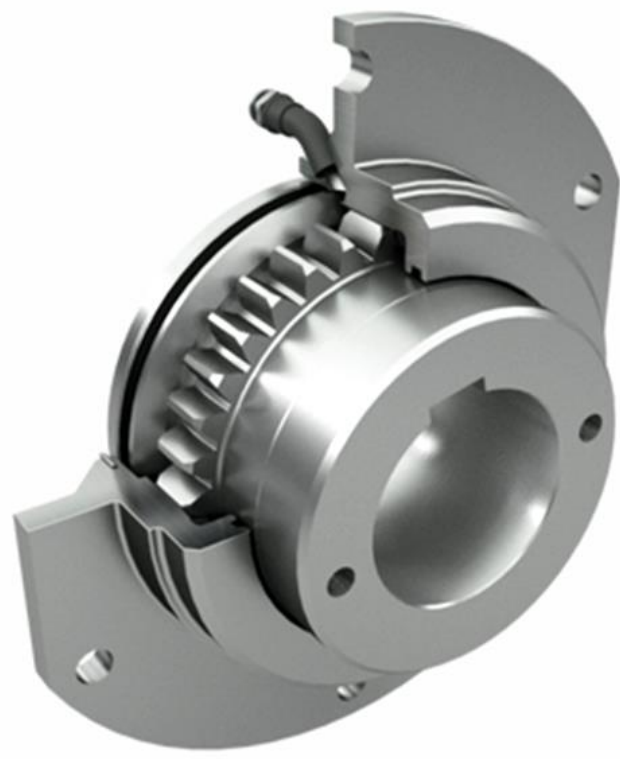

(c)

Fig. 1. Kinematic pairs of different kinds in design of gearing: (a) point-contact kinematic pair in the design of skew-axes gearing, (b) line-contact kinematic pair in the design of helical gearing, and (c) surface-to-surfacecontact kinematic pair in the design of gear coupling.

The beginning of investigation of kinematic pairs can be traced back to the 1875 research undertaken by Franz Reuleaux [20]. Franz Reuleaux introduced the kinematic pair as a new approach to the study of machines that provided an advance over the motion of elements consisting of simple machines [23]. Reuleaux was the first to introduce kinematic pair as a new approach to the study of machines that provided an advance over the motion of elements consisting of simple machines [23].

According to Reuleaux:

Definition 1. Kinematic pair is a connection between two bodies that imposes constraints on their relative movement

In the nowadays terminology the definition to the term kinematic pair is formulated in a slightly different way, namely:

Definition 2. Kinematic pair is a combination of two contiguous links, allowing their relative movement

The following definitions are recommended by IFToMM:

Definition 3. Kinematic pair is a connection between two links restricting their motion

Definition 4. Lower kinematic pair is a kinematic pair that is formed by surface contact between its elements

Definition 5. Higher kinematic pair is a kinematic pair that is formed by either line, or point contact between the elements 


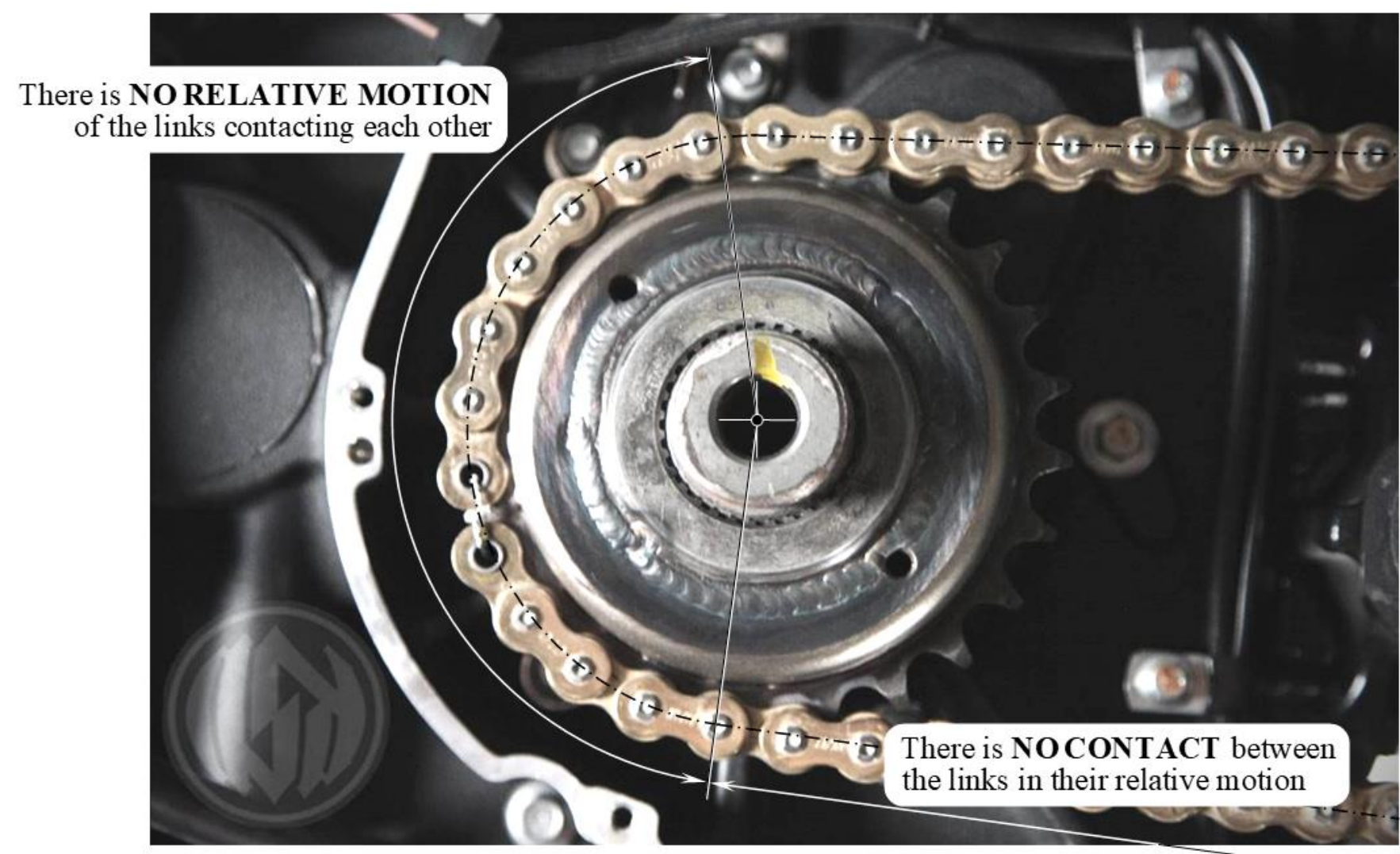

Fig. 2. The so-called wrapping pair (pulley-and-belt drive, chain drive, and so forth) is NOT a kind of kinematic pair, as it does not align to the Reuleaux definition for the term kinematic pair.

In particular, Reuleaux stressed on that kinematic pair consists exactly of two elements, and that these two elements interact with one another ${ }^{2}$. In the matter of connections between rigid bodies, Reuleaux recognized only two kinds of kinematic pairs. He called them higher kinematic pairs and lower kinematic pairs of elements. With higher kinematic pairs, the two elements are in contact at a point, or along a line. Lower kinematic pairs are those featuring surface-to-surface contact. On the premise of this approach numerous classifications of kinematic pairs are developed by the researchers to this end. An example of such classifications of kinematic pairs is illustrated in Fig. 3 [10]. A plurality of variations of this classification can be easily found out in the public domain. No one of these classifications can be referred to as the scientific classification of kinematic pairs. As it follows from the consideration below, in the meantime it is not sufficient to distinguish only lower kinematic pairs with surface-to-surface contact, and higher kinematic pairs that feature either line contact, or point contact of the functional surfaces, like it was acceptable in the time of Franz Reuleaux. There are a few important reasons for that.

\footnotetext{
${ }^{2}$ According to the definition of the term kinematic pair, only two rigid bodies in contact are considered in this text. Further discussion falls into two scenarios.

First, any pair (including, but not limited to kinematic pair) is always comprised of two, and not of three (or more) components. Therefore, two bodies connected by a flexible body (by a belt, a chain, and so forth), do not comprise a kinematic pair in conventional meaning of this term, as three, and not two bodies are involved into the consideration in this case.

Second, if a rigid body and a flexible body are considered kinematic pair as illustrated in Fig. 2, two different configurations of a flexible body and a rigid body are always observed: (a) There is no relative motion of the flexible body in relation to the pulley where these two components contact one another; and (b) There is no contact between the belt and the pulley, where the relative motion of the bodies is observed. In mechanisms with a flexible body there is no relative motion of a pulley and a mating flexible body at points of their contact, that is, they are motionless in relation to one another (see Fig. 2). No contact is observed at the rest of points, at which the pulley and the flexible body travel in relation to each other. Mechanisms with flexible bodies have to be considered separately of kinematic pairs, as such mechanisms are not kinematic pairs by nature.

The so-called "multiple-contacts kinematic pair" is another consideration: Two rigid bodies can make contact at a few points/lines (compound joints). Each of such a contact have to be considered as separate point-contact kinematic pair (or line-contact kinematic pair), as a conventional kinematic pair, and not in whole as a multiple-contacts kinematic pair (see [21], and others).
} 


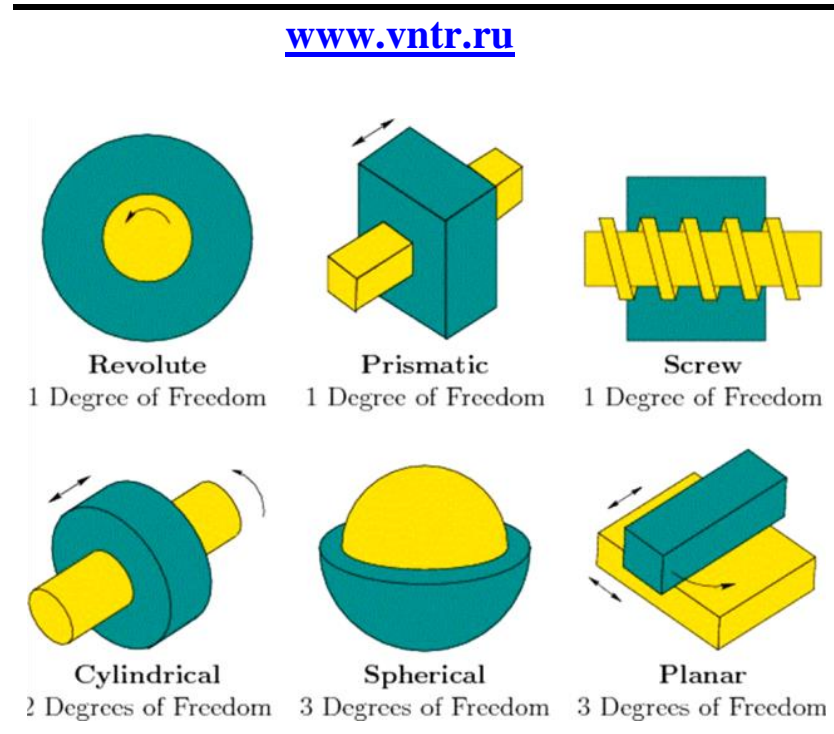

Fig. 3. An example of conventional classification of kinematic pairs. (Adapted from [21]).

№ 161, 2021

First, higher kinematic pairs with point contact, and those with line contact of the functional surfaces, are not equivalent to one another. Therefore, they must be distinguished from one another, and, thus, have to be considered separately. Also, different terms are required to be introduced to refer to higher kinematic pairs with point contact, and to those with line contact of the functional surfaces.

Second, even when the functional surfaces of two kinematic pairs, both, feature either point, or line contact, the performance of kinematic pairs with the same kind of contact can be significantly different depending on the parameters of contact geometry of the functional surfaces. In other words, the performance of two kinematic pairs with point contact (or of two kinematic pairs with line contact) is significantly affected by contact geometry of their functional surfaces, and by the degree of their conformity in particular. The latter is vital (a) for gearing [19] in a general sense, and (b) for high-conformal gearing in particular [15], (c) for contact and bending strength of kinematic pairs; (d) for functional surfaces wear; (e) for elastohydrodynamic lubrication (EHD-lubrication) of heavily-loaded kinematic pairs [22], and so forth.

The outlined features of kinematic pairs with point, and with line contact of functional surfaces, along with a few more features mentioned below, reveal the necessity in a more in-depth investigation into possible kinds of kinematic pairs for the needs of the nowadays industry.

2. Conventional Approach to Design and Investigation of Kinematic Pairs. When kinematic pairs are discussed, most sources available in the public domain provide mainly several kinematic pair schemes. Plane-to-plane, ball in a pocket, round cylinder-to-plane, ball-to-plane, and so forth, are among them. The total number of designs of kinematic pairs, considered in the textbooks on theory of machines and mechanisms, does not exceed a dozen or so. No comprehensive and systematic analysis of all possible kinds of kinematic pairs has been undertaken to this end.

Kinematic synthesis of linkages was carried out by Denavit and Hartenberg [1]. An extensive research on possible kinds of kinematic pairs was undertaken by Dr. L.T. Dvornikov [5], who also worked on the development of the theory of kinematic pairs [6]. Many efforts on the development of the theory and systematization of kinematic pairs of mechanical systems were undertaken by Dr. E.Ya. Zhivago [24], as well as numerous of others.

Without going into details of the analysis of the results of the research achieved to this end, a following conclusion can be drawn up:

Conclusion 1. The theory of kinematic pairs is too far from to be considered complete.

Therefore, a more in detail research into possible kinds of kinematic pairs, and the development of their scientific classification is required to be undertaken.

Following the terminology adopted since the time of $F$. Reuleaux, higher kinematic pairs are comprised of two functional surfaces that make contact at point. Therefore, two functional surfaces in point contact are sufficient to specify a corresponding higher kinematic pair. However, a plurality of kinematic pairs that meet the specified requirements (two surfaces that make point contact), may feature significantly different performance. This means that the conventional definition of the term higher kinematic pair is insufficient as it doesn't establish one-to-one correspondence between a particular kinematic pair and its anticipated performance. It is evident 
that, for example, convex-to-convex contact of two functional surfaces, and their convex-to-concave contact significantly different from one another from the perspective of bearing capacity, regardless of that in both cases the functional surfaces make point contact, and in both cases, they are referred to as higher kinematic pair. This practice is no longer acceptable.

Another example of two higher kinematic pairs that, from the first glimpse, appear very similar (or almost identical) to one another pertains to convex-to-concave contact of two functional surfaces with a small difference between radii of their normal curvature. When the difference between the radii of normal curvature, in one of two kinematic pairs significantly smaller than that in the other kinematic pair, the bearing capacity of the first one is drastically different from that of the second one (again, regardless of both the kinematic pairs are still referred to as higher kinematic pair).

Inconsistency of the adopted terminology in the realm of higher kinematic pairs is clearly illustrated by the two just considered examples. More in detail analysis of contact geometry of two functional surfaces in a higher kinematic pair is necessary to be undertaken in order to better understand all the features and behavior of kinematic pairs of different design.

An abridged version of the developed scientific classification of kinematic pairs is discussed below in this paper. The interested reader may wish to go to [16] where full version of this classification is disclosed.

3. Instantaneous Kinematics (Mobility) of Kinematic Pairs. It is adopted below in this paper that a kinematic pair that features a highest possible degree of freedom is referred to as the most general one.

A kinematic pair of five degrees of freedom (five DoF kinematic pair) is schematically shown in Fig. 4. Here, two bodies, namely, a Body 1, and a Body 2 , are bounded by smooth regular surfaces. The bodies, $B_{1}$ and $B_{2}$, make contact at point $K$ . The origin of a local reference system, $x_{K} y_{K} z_{K}$ , coincides with the contact point, $K$. The coordinate axes, $x_{K}, y_{K}$, and $z_{K}$, are along the corresponding unit vectors $\mathbf{t}_{1 . B_{1}}, \mathbf{t}_{1 . B_{2}}$, and $\mathbf{n}_{B_{1}}$, of the Darboux frame, $\mathbf{t}_{1 . B_{1}} \mathbf{t}_{1 . B_{2}} \mathbf{n}_{B_{1}}$. Depending on timing of the elementary linear and rotary motions $\mathbf{v}_{x}$ and $\boldsymbol{\omega}_{x}$, as well as of $\mathbf{v}_{y}$ and $\boldsymbol{\omega}_{y}$, four motions of the Body 2 over the Body 1 are permissible:

- Sliding over the Body 1 (with the contact point trace within the plane $y_{K} z_{K}$ )

- Rolling over the Body 1 (with the contact point

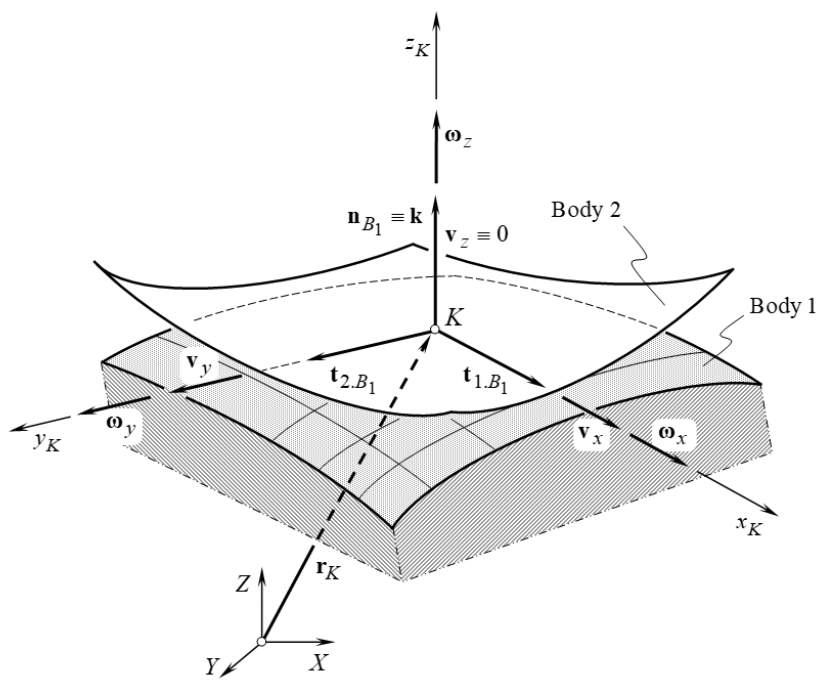

Fig. 4. Schematic of five degree-of-freedom kinematic pair. trace within the plane $y_{K} z_{K}$ )

- Sliding over the Body 1 (with the contact point trace within the plane $x_{K} z_{K}$ )

- Rolling over the Body 1 (with the contact point trace within the plane $x_{K} z_{K}$ )

The rotation, $\boldsymbol{\omega}_{z}$, of the Body 2 about the common perpendicular, $\mathbf{n}_{B_{1}}$, is the fifth permissible motion of the Body 2 in relation to the Body 1.

Point-contact kinematic pairs may feature up to five degrees of freedom. Kinematic pairs that feature surface-to-surface contact between the functional surfaces, feature not more than three degrees of freedom. Therefore, from the standpoint of mobility, point-contact kinematic pairs are more general compared to surface-to-surface contact kinematic pairs. Line-contact kinematic pairs 


\section{Www.vntr.ru}

№ 161, 2021

occupy a position between the point-contact kinematic pairs, and between surface-to-surface contact kinematic pairs.

The kinematic pairs with a lesser mobility than that shown in Fig. 4, can be viewed as a reduced case of point-contact kinematic pair.

4. Contact Geometry of Functional Surfaces in Kinematic Pairs. The problem of analytical description of contact geometry of two smooth regular surfaces in the first order of tangency is a challenging one. Contact geometry of curves and surfaces is under consideration by scientists for a long while. Investigation of contact geometry of curves and surfaces can be traced back to the eighteenth century. The study of the contact of curves and surfaces was undertaken in considerable detail by J.L. Lagrange in his "Theorié des Fonctions Analytiques" (1797) [10], and A.L. Cauchy in his "Leçons sur les Applications du Calcul Infinitésimal á la Geometrie" (1826) [1]. Later on, in the twentieth century, an investigation in the realm of contact geometry of curves and surfaces was undertaken by J. Favard in his "Course de Gèomètrie Diffèrentialle Locale" (1957) [8]. A few more names of researchers in the field are to be mentioned.

4.1. Dupin indicatrix at point of functional surface. Various methods for analytical description of contact geometry between two smooth regular surfaces have been developed. An overview of the methods can be found out in the monograph by Prof. S.P. Radzevich [18]. Latest achievements in the field are discussed in the paper [17] and others, and in the monographs [13] and [14].

An earlier developed powerful method for analytical description of contact geometry of two functional surfaces in a kinematic pair is based on the geometry of Dupin indicatrices, Dup $\left(B_{1}\right)$ and $\operatorname{Dup}\left(B_{2}\right)$, at a point of contact of functional surfaces $B_{1}$ and $B_{2}$, correspondingly.

In a common section of two functional surfaces, $B_{1}$ and $B_{2}$, by a normal plane, consider a unit tangent vector, $\mathbf{t}_{c n f}(\varphi)$, through a contact point, $K$. The unit tangent vector, $\mathbf{t}_{c n f}(\varphi)$, is entirely located in a common tangent plane through $K$. In a common normal section, the radii of curvature of the functional surfaces, $B_{1}$ and $B_{2}$, are designated as $R_{B_{1}}$ and $R_{p}$, correspondingly. Let's designate $r_{B_{1}}(\varphi)=\sqrt{R_{B_{1}}(\varphi)}$, and $r_{p}(\varphi, \mu)=\sqrt{R_{p}(\varphi, \mu)}$.

By definition, position vector of point, $\mathbf{r}_{B_{1}}$, of the Dupin indicatrix, $\operatorname{Dup}\left(B_{1}\right)$, is specified as:

$$
\operatorname{Dup}\left(B_{1}\right) \Rightarrow \mathbf{r}_{B_{1}}(\varphi)=\sqrt{R_{B_{1}}(\varphi)} \cdot \mathbf{t}_{B_{1}}(\varphi)
$$

At point of a planar local patch of a functional surface, $B$, an inverse Dupin indicatrix, $\operatorname{Dup}_{k}(B)$ [and a corresponding inverse curvature indicatrix, $\operatorname{Crv}_{k}(B)$ ], can be used instead of conventional $\operatorname{Dup}(B)$ [and instead of $\operatorname{Crv}(B)]$.

In a planar local patch of a functional surface, $B$, the inverse Dupin indicatrix, $\operatorname{Dup}_{k}(B)$, shrinks to the contact point, $K$.

Here, in Eq. (1), $\mathbf{t}_{B_{1}}$ is the unit tangent vector through the contact point, $K$ [in its current configuration, the vector $\mathbf{t}_{B_{1}}$ forms an angle, $\varphi$, with the first principal direction, $\mathbf{t}_{1 . B_{1}}$, on the functional surface $B_{1}$, that is $\left.\varphi=\angle\left(\mathbf{t}_{B_{1}}, \mathbf{t}_{1 . B_{1}}\right)\right]$.

Two examples of Dupin indicatrices along with curvature indicatrices [here and below, curvature indicatrix, $\operatorname{Crv}\left(B_{1}\right)$, at a surface point is a portion of tangent plane bounded by Dupin indicatrix, $\left.\operatorname{Dup}\left(B_{1}\right)\right]$ at point of smooth regular functional surfaces are illustrated in Fig. 5. Note that curvature indicatrices are not labeled here, in Fig. 5. In total, there are ten different kinds of

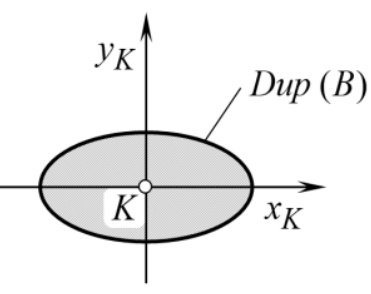

Convex elliptical

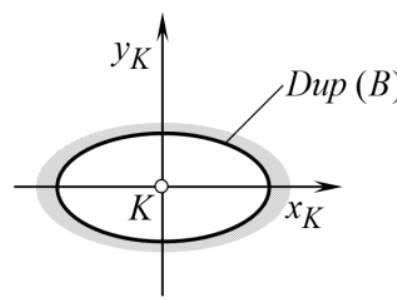

Concave elliptical

Fig. 5. Examples of Dupin indicatrices, Dup (B), and curvature indicatrices, $\operatorname{Crv}(B)$, at elliptical point of functional surface, $B$. local patches of the functional surfaces, and, 
therefore, there are just ten different kinds of the corresponding Dupin indicatrices [16]. Here, in Fig. 5, functional surfaces are designated as $B$.

4.2. Indicatrix of conformity at point of contact of functional surfaces. For the analytical description of contact geometry of functional surfaces, indicatrix of conformity, $\operatorname{Cnf}_{R}\left(B_{1} / B_{2}\right)$, at point of contact of the bodies $B_{1}$ and $B_{2}$ can be used. The construction of this planar characteristic curve, $\operatorname{Cnf}_{R}\left(B_{1} / B_{2}\right)$, of the fourth order is based on Dupin indicatrices, Dup $\left(B_{1}\right)$ and $\operatorname{Dup}\left(B_{2}\right)$.

Position vector, $\mathbf{r}_{c n f}$, of point of indicatrix of conformity ${ }^{3}, C_{n f}\left(B_{1} / B_{2}\right)$ [11], [12], at point of contact of two functional surfaces, $B_{1}$ and $B_{2}$, can be expressed in terms of the distances $r_{B_{1}}$ and $r_{B_{2}}$ as follows (see Fig. 6):

$$
\operatorname{Cnf}_{R}\left(B_{1} / B_{2}\right) \Rightarrow \mathbf{r}_{c n f}(\varphi, \mu)=\left[r_{B_{1}}(\varphi)-r_{B_{2}}(\varphi+\mu)\right] \cdot \mathbf{t}_{c n f}(\varphi)
$$

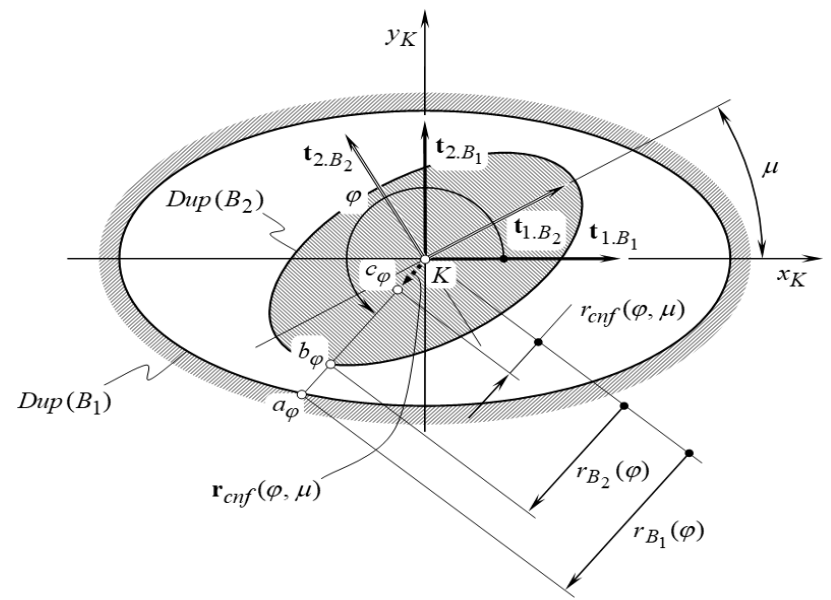

Fig. 6. On derivation of equation of indicatrix of conformity, $\operatorname{Cnf}_{R}\left(B_{1} / B_{2}\right)$, at point of contact, $K$, of two functional surfaces $B_{1}$ and $B_{2}$.

The functional surfaces are turned in relation to one another about the common perpendicular, $\mathbf{n}_{B_{1}}$, through an angle. This angle, $\mu$, is referred to as the angle of the surfaces' local relative orientation, $\mu$. The angle $\mu$, is formed by the unit vectors of the first principal directions, $\mathbf{t}_{1 . B_{1}}$ and $\mathbf{t}_{1 . B_{2}}$ (or, the same, of the second principal directions, $\mathbf{t}_{2 . B_{1}}$ and $\mathbf{t}_{2 . B_{2}}$ ), that is, $\mu=\angle\left(\mathbf{t}_{1 . B_{1}}, \mathbf{t}_{1 . B_{2}}\right)$ [or $\mu=\angle\left(\mathbf{t}_{2 . B_{1}}, \mathbf{t}_{2 . B_{2}}\right)$ ].The unit vector, $\mathbf{t}_{c n f}(\varphi)$, can be expressed in terms of fundamental magnitudes of the first and the second order calculated at point of contact, $K$, of the functional surfaces, $B_{1}$ and $B_{2}$.

The interested reader is referred to [13], [14], [15], [16], [19], as well as to other advanced sources, for more details on the indicatrix of conformity at point of contact between two

smooth regular surfaces.

Dupin indicatrices, $\operatorname{Dup}\left(B_{1}\right)$ and $\operatorname{Dup}\left(B_{2}\right)$, along with indicatrices of conformity, $C n f_{R}\left(B_{1} / B_{2}\right)$, at point of contact, $K$, of two functional surfaces, $B_{1}$ and $B_{2}$, are extensively used below for the analysis of contact geometry of interacting surfaces, $B_{1}$ and $B_{2}$.

5. Kinds of Functional Surfaces. The total number of different surfaces that bound real objects is infinitely large. A systematic consideration of surfaces for the purposes of investigation and analysis of kinematic pairs is of critical importance. Therefore, in order to proceed with the analysis of contact geometry of functional surfaces in kinematic pairs, an in-detail analysis of possible kinds of functional surfaces is required to be performed.

The total number of possible kinds of functional surfaces in kinematic pairs actually is endless. An advantage can be taken from that in kinematic pairs of the most general kinds only small portions of

\footnotetext{
${ }^{2}$ For the first time ever an equation of the indicatrix of conformity, $\operatorname{Cnf}_{R}\left(B_{1} / B_{2}\right)$, was published in:

Pat. No. 1,185,749, USSR, A Method of Sculptured Surface Machining on a Multi-Axis NC Machine./S.P. Radzevich, Int. Cl. B23c 3/16, Filed: October 24, 1983.

Pat. No. 1,249,787, USSR, A Method of Sculptured Surface Machining on a Multi-Axis NC Machine./S.P. Radzevich, Int. Cl. B23c 3/16, Filed: December 27, 1984.
} 
www.vntr.ru

№ 161, 2021

the entire functional surfaces interact with one another. It is common practice to refer to small portions of functional surfaces as to the local patches of functional surface. At point of a local patch of every functional surface two principal radii of curvature, $R_{1}$ and $R_{2}$ (here an equality, $R_{2}>R_{1}$, is always valid), can be specified. The principal radii of curvature, $R_{1}$ and $R_{2}$, are signed values: they are positive for convex, are negative for concave, and are equal to a zero for flatten section of a surface by principal planes, $C_{1}$ and $C_{2}$. No inequality of the kind $R_{2} \geq R_{1}$ is permissible, as in the case of $R_{2}=R_{1}$ no principal directions at a surface point can be identified).

Mean curvature, $M$, and Gaussian curvature, $G$, can be attributed to every surface point:

$$
\begin{gathered}
M=\frac{R_{1}+R_{2}}{2} \\
G=R_{1} \cdot R_{2}
\end{gathered}
$$

Different kinds of local patches of functional surfaces are distinguished based on various permissible combinations of the actual values of the mean curvature, $M:(M>0, M<0, M=0$ ), and the total curvature, $G: G>0, G<0, G=0$, at surface point. At umbilic surface point, an equality, $M=k$, is valid (here the normal curvature at the umbilic surface point is denoted by $k$ ).

The total number of local patches of functional surface is limited just to 10 possible their kinds [13], [14], [16]. They are as follows:

1. Three kinds of convex, namely, with a positive Gauss curvature ( $G>0$ ), and a positive mean curvature $(M>0)$ local patches of functional surfaces

2. Three kinds of concave, namely, with a positive Gauss curvature $(G>0)$, and a negative mean curvature $(M<0)$ local patches of functional surfaces

3. Three kinds of hyperbolic, namely, with a negative Gauss curvature $(G<0)$, and either with a positive $(M>0)$, or with a negative $(M<0)$ mean curvature local patches of functional surfaces

4. One kind of a flatten, namely, with a zero Gauss curvature $(G=0)$, and either with a positive ( $M \rightarrow+\infty)$, or with a negative $(M \rightarrow-\infty)$ mean curvature local patches of functional surfaces. These two kinds of flatten local patches of functional surfaces (either with $M \rightarrow+\infty$, or with $M \rightarrow-\infty)$ are equivalent to one another.

Dupin indicatrices, Dup $(B)$, along with curvature indicatrices, $\operatorname{Crv}(B)$, for all possible kinds of local patches of functional surfaces in kinematic pairs are depicted in Fig. 5.

For a planar local patch of the functional surface $B$, points of the curvature indicatrices $\operatorname{Crv}(B)$ either occupy all the plane surface, or all of them are remote to infinity. Therefore, no graphical interpretation is provided to the curvature indicatrices $\operatorname{Crv}(B)$ of a plana local patch of the functional surface in kinematic pairs.

6. Kinds of Kinematic Pairs. On the premise of the definition to the term kinematic pair (Reuleaux, 1875): kinematic pair is a connection between two bodies that imposes constraints on their relative movement, a following approach is adopted in this paper to develop a scientific classification of kinematic pairs.

A kinematic pair that features a highest possible degree of freedom corresponds to the first stratus in the classification. The fewer the degree of freedom, the lower the stratus of a kinematic pair in the classification, and vice versa.

In the rest of the text below, the terms higher kinematic pair and lower kinematic pair, and are not used at all. Instead, the following kinds of kinematic pairs are distinguished:

1. Point-contact kinematic pairs

2. Line-contact kinematic pairs

3. Surface-to-surface-contact kinematic pairs 
Kinematic pairs of all kinds fall into one of these categories.

\begin{tabular}{|c|c|c|c|c|c|c|c|c|c|c|c|c|}
\hline \multirow{4}{*}{\multicolumn{3}{|c|}{$\begin{array}{l}\text { Local patch of functional } \\
\text { surface } B_{1}\end{array}$}} & \multicolumn{10}{|c|}{ Local patch of functional surface $B_{2}$} \\
\hline & & & \multicolumn{4}{|c|}{ Elliptic, ${ }^{2} Q>0 ;{ }^{2} \mathscr{C} \neq 0$} & \multicolumn{3}{|c|}{ Parabolic, ${ }^{2}=0$} & \multicolumn{3}{|c|}{ Hyperbolic, ${ }^{2} \&<0$} \\
\hline & & & \multicolumn{2}{|c|}{${ }^{2} \mathscr{C}>0$} & \multicolumn{2}{|c|}{$2 \mathscr{C H}<0$} & \multirow{2}{*}{$2_{\mathscr{C H}}>0$} & \multirow{2}{*}{${ }^{2} \mathscr{C}=0$} & \multirow{2}{*}{$2_{\mathscr{U}}<0$} & \multirow{2}{*}{$2_{\mathscr{C}}>0$} & \multirow{2}{*}{${ }^{2} \mathscr{C}=0$} & \multirow{2}{*}{${ }^{2} \mathscr{C O C}<0$} \\
\hline & & & ${ }^{2} \mathscr{C R}>0$ & $2 \mathscr{\mathscr { C }}={ }^{2} k$ & ${ }^{2} \mathscr{G C}<0$ & ${ }^{2} \mathscr{G C}={ }^{2} k$ & & & & & & \\
\hline \multirow{4}{*}{ 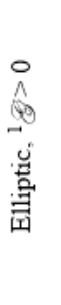 } & \multirow{2}{*}{$1_{\mathscr{C}}>0$} & $1_{\mathscr{C}}>0$ & 1.1 & 1.2 & 1.3 & 1.4 & 1.5 & 1.6 & 1.7 & 1.8 & 1.9 & 1.10 \\
\hline & & $1_{\mathscr{C O}}={ }^{1} k$ & 2.1 & 2.2 & 2.3 & 2.4 & 2.5 & 2.6 & 2.7 & 2.8 & 2.9 & 2.10 \\
\hline & \multirow{2}{*}{$1_{\mathscr{C}}<0$} & $1_{\mathscr{L}}<0$ & 3.1 & 3.2 & 3.3 & 3.4 & 3.5 & 3.6 & 3.7 & 3.8 & 3.9 & 3.10 \\
\hline & & $1_{\mathscr{C} C}={ }^{1} k$ & 4.1 & 4.2 & 4.3 & 4.4 & 4.5 & 4.6 & 4.7 & 4.8 & 4.9 & 4.10 \\
\hline \multirow{3}{*}{ 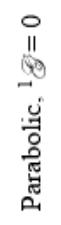 } & \multicolumn{2}{|c|}{$1_{\mathscr{C}}>0$} & 5.1 & 5.2 & 5.3 & 5.4 & 5.5 & 5.6 & 5.7 & 5.8 & 5.9 & 5.10 \\
\hline & \multicolumn{2}{|c|}{$1_{\mathscr{S}}=0$} & 6.1 & 6.2 & 6.3 & 6.4 & 6.5 & 6.6 & 6.7 & 6.8 & 6.9 & 6.10 \\
\hline & \multicolumn{2}{|c|}{$1_{\mathscr{S}}<0$} & 7.1 & 7.2 & 7.3 & 7.4 & 7.5 & 7.6 & 7.7 & 7.8 & 7.9 & 7.10 \\
\hline \multirow{3}{*}{ 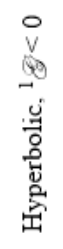 } & \multicolumn{2}{|c|}{$1_{\mathscr{C}}>0$} & 8.1 & 8.2 & 8.3 & 8.4 & 8.5 & 8.6 & 8.7 & 8.8 & 8.9 & 8.10 \\
\hline & \multicolumn{2}{|c|}{$1_{\mathscr{C}}=0$} & 9.1 & 9.2 & 9.3 & 9.4 & 9.5 & 9.6 & 9.7 & 9.8 & 9.9 & 9.10 \\
\hline & \multicolumn{2}{|c|}{$1_{\mathscr{C}}<0$} & 10.1 & 10.2 & 10.3 & 10.4 & 10.5 & 10.6 & 10.7 & 10.8 & 10.9 & 10.10 \\
\hline
\end{tabular}

Fig. 7. Morphological matrix $10 \times 10$ with all permissible kinds of contact of the functional surfaces $B_{1}$ and

$B_{2}$ in a kinematic pair.

Taking into account that there are only ten different kinds of local patches of smooth, regular functional surfaces $B_{1}$ and $B_{2}$ (see Fig. 5), every kind of the surfaces contact can be represented more in detail. For the analysis, a square morphological matrix of size $10 \times 10=100$ is composed. An example of the morphological matrix is illustrated in Fig. 7. All possible combinations of the surfaces contact are covered by this morphological matrix. One axis of the morphological matrix is represented by ten kinds of local patches of the functional surface $B_{1}$, while another axis is represented by ten kinds of local patches of the functional surface $B_{2}$. The morphological matrix contains 100 different combinations of the local patches of functional surfaces $B_{1}$, and of functional surfaces $B_{2}$. As some kinds of contact of local patches $B_{1}$ and $B_{2}$ appear twice in the morphological matrix, therefore, only:

$$
C_{10}^{2}+10=\frac{10 !}{2 !(10-2) !}+10=55
$$

of them are necessary to be investigated in detail. For example, no contact is permissible between two concave surfaces $B_{1}$ and $B_{2}$. Also, concave surfaces can't make contact with saddle-like surfaces, and so forth. All of these and the similar combinations of the surfaces $B_{1}$ and $B_{2}$ are also eliminated from the further analysis (see Fig. 7). Ultimately, for point contact kinematic pairs, the total number of contacts between the bodies, $B_{1}$ and $B_{2}$, is limited only to 29 different kinds. 


\section{Www.vntr.ru}

№ 161, 2021

The morphological matrix $10 \times 10$ (see Fig. 7) is used with a goal to perform an in-detail analysis of kinematic pairs. In this matrix, the combinations of the functional surfaces, $B_{1}$ and $B_{2}$, along the "diagonal" formed by the pairs 1.1, 2.2, 3.3, ., 10.10 (see Fig. 7), and below the diagonal are taken into account, while the rest of the combinations of the surfaces $B_{1}$ and $B_{2}$ located above the diagonal, can be found out among those located below the diagonal.

6.1. Point-contact kinematic pairs. In point-contact kinematic pairs (or just $P_{c}$-kinematic pairs, for simplicity), the functional surfaces, $B_{1}$ and $B_{2}$, make contact at a single point. A more in detail analysis reveals that following kinds of contact between the functional surfaces, $B_{1}$ and $B_{2}$, have to be recognized in point-contact kinematic pairs.

6.1.1. True-point-contact kinematic pairs. The true-point-contact kinematic pairs (or just $T P_{c}-$ kinematic pairs, for simplicity) of different kinds are distinguished.

First, there are several different kinds of convex-to-convex true-point-contact kinematic pairs that feature functional surfaces, $B_{1}$ and $B_{2}$, either of elliptic, or of umbilic, or of parabolic geometry. The kinematic pairs of this kind are composed of two convex functional surfaces, $B_{1}$ and $B_{2}$ (see Fig. 8):

- Elliptic-to-elliptic-contact kinematic pair

- Elliptic-to-umbilic-contact kinematic pair

- Elliptic-to-parabolic-contact kinematic pair

- Umbilic-to-umbilic-contact kinematic pair

- Umbilic-to-parabolic-contact kinematic pair

- Parabolic-to-parabolic-contact kinematic pair

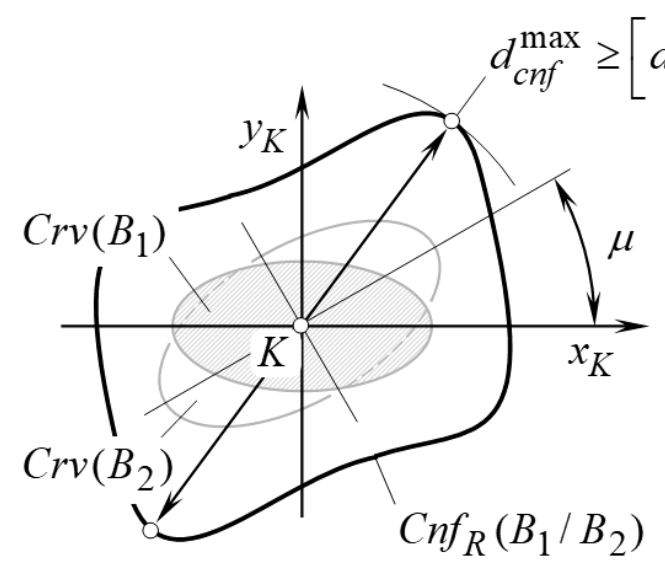

Elliptic-to-elliptic

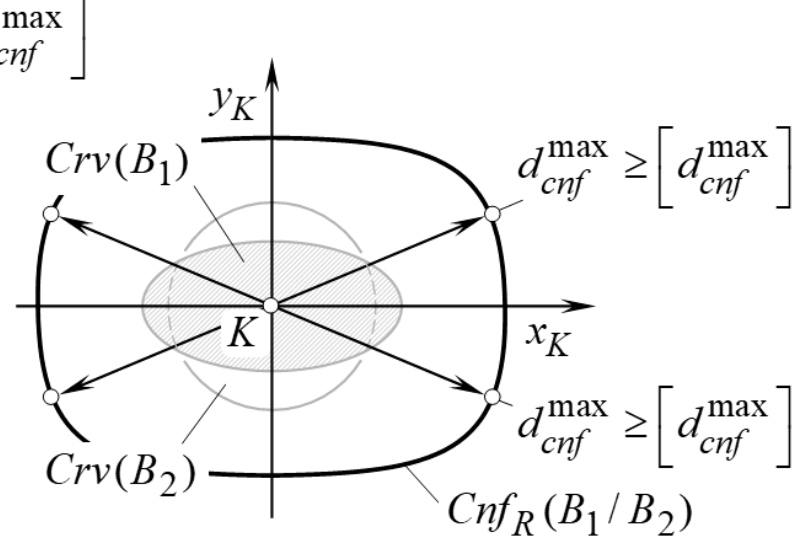

Elliptic-to-umbilic

1.1

Fig. 8. Examples of indicatrices of conformity, $\operatorname{Cnf}_{R}\left(B_{1} / B_{2}\right)$, and curvature indicatrices,

$$
\operatorname{Crv}_{R}\left(B_{1}\right)
$$


The kinematic pairs of this kind feature a single contact point between the functional surfaces $B_{1}$ and $B_{2}$. Two examples of indicatrices of conformity, $\operatorname{Cnf}_{R}\left(B_{1} / B_{2}\right)$, in $T P_{c}$-kinematic pairs (that correspond to 1.1 and 2.1 contacts of the functional surfaces $B_{1}$ and $B_{2}$ in Fig. 7) are shown in Fig. 8. The rest of the kinematic pairs (that correspond to 2.2, 5.1, 5.2, and $\mathbf{5 . 5}$ contacts of the functional surfaces) are discussed in detail in [16].

There are no constraints onto the actual value of the angle of local relative orientation, $\mu$, of the contacting functional surfaces, $B_{1}$ and $B_{2}$, for the kinematic pairs with contact of the kinds 1.1, 2.1,

2.2, 5.1, and 5.2 (see Fig. 7). For the kinematic pair labeled 5.5, the values, $\mu=0^{\circ}$ and $\mu= \pm 180^{\circ}$, of the angle, $\mu$, are not permissible, as in this scenario the kinematic pair $\mathbf{5 . 5}$ is no longer a kind of convex-to-convex true-point-contact kinematic pair.

The diagrams (see Fig. 8), correspond to the so-called non-conformal kinematic pairs. The term non-conformal kinematic pair is due to in all sections of the functional surfaces by a plane through the common perpendicular the lines of intersection of the surfaces make either convex-to-convex, or convex-to-straight line contact (therefore, they are of non-negative values).

Second, there are only two different kinds of convex-to-planar-point-contact kinematic pairs that are composed either of elliptic, or of umbilic functional surface, $B_{1}$, in contact with a planar local patch of a mating functional surface, $B_{2}$. Contact characteristics of these kinematic pairs are shown in Fig. 9:

The kinematic pairs of this kind are composed of two functional surfaces, $B_{1}$ and $B_{2}$ :

- Planar-to-elliptic-contact kinematic pair

- Planar-to-umbilic-contact kinematic pair

Kinematic pairs of this kind feature a single contact point between the functional surfaces $B_{1}$ and $B_{2}$. In Fig. 7 , these kinematic pairs correspond to $\mathbf{6 . 1}$, and $\mathbf{6 . 2}$, contacts of the functional surfaces $B_{1}$ and $B_{2}$. The cases 6.5, and $\mathbf{6 . 6}$ are not considered here as the kinematic pairs of these kinds are not true-pointcontact kinematic pairs. There are no constraints onto the actual value of the angle of local relative orientation, $\mu$, of the functional surfaces, $B_{1}$ and $B_{2}$, for the kinematic pairs labeled 6.1, and 6.2.

Third, there are several different kinds of convex-to-hyperbolic-point-contact

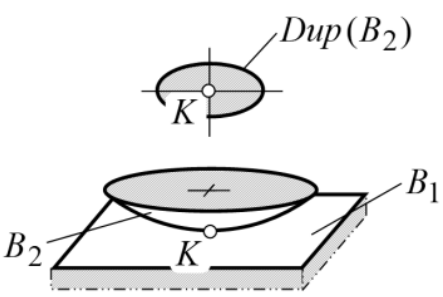

Elliptic-to-plane

6.1

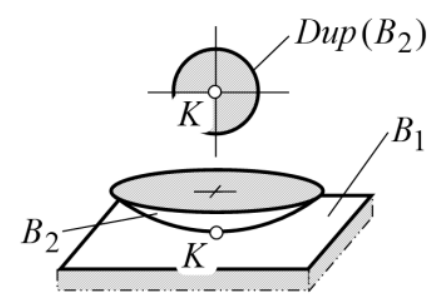

Umbilic-to-plane

6.2

Fig. 9. Features of point-contact kinematic pairs composed of a planar functional surface $B_{1}$ and either of elliptic, or umbilic functional surface $B_{2}$. kinematic pairs that are comprised of different kinds of hyperbolic functional surface, $B_{1}$, in contact with convex local patches of a mating functional surface, $B_{2}$. In Fig. 7, these kinematic pairs correspond to 8.1, 8.2, 9.1, 9.2, 10.1, and 10.2, contacts of the functional surfaces $B_{1}$ and $B_{2}$, (see Fig. 10):

- Elliptic-to-hyperbolic (pseudo-concave)-contact kinematic pair

- Umbilic-to-hyperbolic (pseudo-concave)-contact kinematic pair

- Elliptic-to-minimal (pseudo-umbilic)-contact kinematic pair

- Umbilic-to-minimal (pseudo-umbilic)-contact kinematic pair

- Elliptic-to-hyperbolic (pseudo-convex)-contact kinematic pair 
www.vntr.ru

№ 161, 2021

- Umbilic-to-hyperbolic (pseudo-convex)-contact kinematic pair

In kinematic pairs with contacts of the kinds 8.1, 9.1, and 10.1, the actual value of the angle, $\mu$, of the functional surfaces local relative orientation is in the range of: $\mu_{\min }<\mu<\mu_{\max }$, where $\mu_{\min }$ and $\mu_{\max }$ are the minimum and the maximum permissible values of the angle $\mu$, correspondingly.

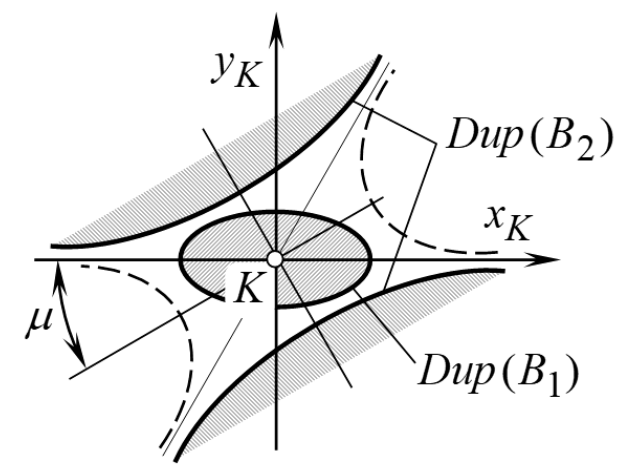

Elliptic-to-hyperbolic (pseudo-concave)

\section{1}

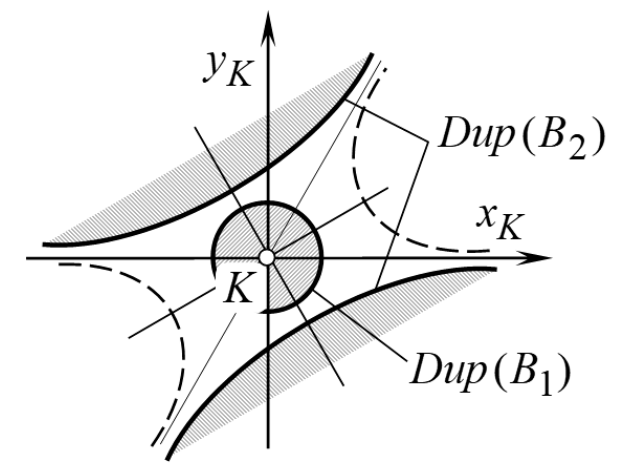

Umbilic-to-hyperbolic (pseudo-concave)

8.2

Fig. 10. Indicatrices of conformity, $\operatorname{Cnf}_{R}\left(B_{1} / B_{2}\right)$, and the Dupin indicatrices, $\operatorname{Dup}\left(B_{1}\right)$ and $\operatorname{Dup}\left(B_{2}\right)$, at contact point of kinematic pairs that feature convex-to-saddle-like point-contact of the functional surfaces $B_{1}$ and $B_{2}$.

There are no constraints onto the actual value of the angle of local relative orientation, $\mu$, of the functional surfaces, $B_{1}$ and $B_{2}$, for the kinematic pairs with contacts of the kinds 8.2, 9.2, and 10.2. The diagrams, shown in Fig. 10, correspond to the so-called semi-conformal kinematic pairs. The term semi-conformal kinematic pair is due to in certain sections of the functional surfaces by a plane through the common perpendicular the lines of intersection of the surfaces make either convex-to-convex, or concave-to-convex contact (that is, curvature of the curves of intersection of the functional surfaces are of opposite sign).

Fourth, there are three different kinds of convex-to-hyperbolic-point-contact kinematic pairs that are composed of a hyperbolic functional surface, $B_{1}$, in contact with convex parabolic local patches of a mating functional surface, $B_{2}$. In Fig. 7, kinematic pairs of this kind correspond to 8.5, 9.5, and 10.5, contacts of the functional surfaces $B_{1}$ and $B_{2}$ (see Fig. 11):

- Parabolic-to-hyperbolic (pseudo-concave)-contact kinematic pair

- Parabolic-to-minimal (pseudo-umbilic)-contact kinematic pair

- Parabolic-to-hyperbolic (pseudo-convex)-contact kinematic pair

For the kinematic pairs of these three kinds, the actual value of the angle, $\mu$, of the functional surfaces local relative orientation is in the range of: $\mu_{\min }<\mu<\mu_{\max }$.

To a certain extent, the diagrams in Fig. 11 can be considered as semi-conformal kinematic pairs. In certain sections of the functional surfaces by a plane through the common perpendicular the lines of intersection of the surfaces make either straight-to-convex, or concave-to-convex contact.

Fifth, there are three different kinds of hyperbolic-to-hyperbolic-point-contact kinematic pairs that are composed of different kinds of hyperbolic functional surfaces, $B_{1}$, in contact with hyperbolic 
local patches of a mating functional surface, $B_{2}$. In Fig. 7, kinematic pairs of this kind correspond to 10.8, 10.9, and 10.10, contacts of the functional surfaces $B_{1}$ and $B_{2}$ (see Fig. 12):

- Hyperbolic (pseudo-convex)-to-hyperbolic (pseudo-concave)-contact kinematic pair

- Hyperbolic (minimal)-to-hyperbolic (pseudo-concave)-contact kinematic pair

- Hyperbolic (pseudo-concave)-to-hyperbolic (pseudo-concave)-contact kinematic pair

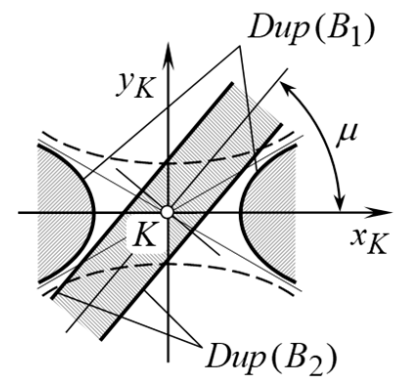

Parabolic-to-hyperbolic (pseudo-convex)

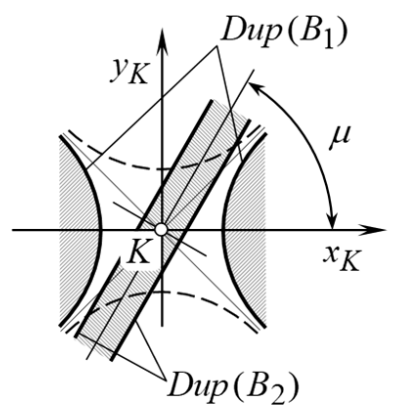

Parabolic-to-minimal (pseudo-umbilic)

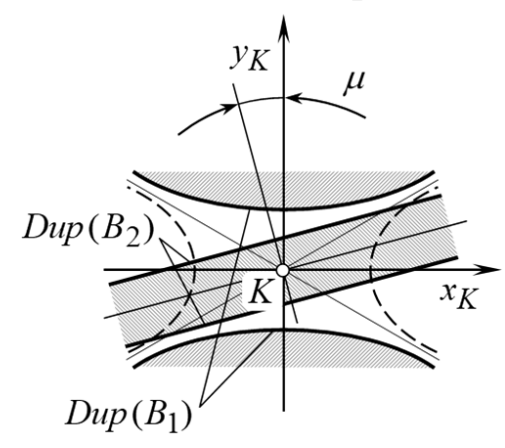

Parabolic-to-hyperbolic (pseudo-concave)

8.5

9.5

10.5

Fig. 11. Relative orientation of the Dupin indicatrices, $\operatorname{Dup}\left(B_{1}\right)$ and $\operatorname{Dup}\left(B_{2}\right)$, at contact point of true-point-contact kinematic pairs that feature convex-parabolic-to-hyperbolic contacts of the functional surfaces $B_{1}$ and $B_{2}$ (the actual values of the angle, $\mu$, of the functional surfaces, $B_{1}$ and $B_{2}$, local relative orientation is in the range of: $\mu_{\min } \leq \mu \leq \mu_{\max }$ ).

For the kinematic pairs of these three kinds, the actual value of the angle, $\mu$, of the functional surfaces local relative orientation is in the range of: $\mu_{\min }<\mu<\mu_{\max }$.

The shown in Fig. 12 diagrams correspond to the so-called semi-conformal kinematic pairs.

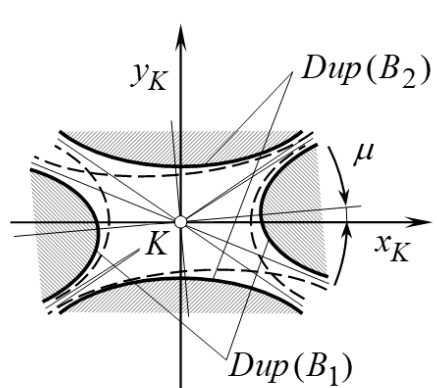

Hyperbolic (pseudo-convex)-to hyperbolic-(pseudo-concave)

10.8

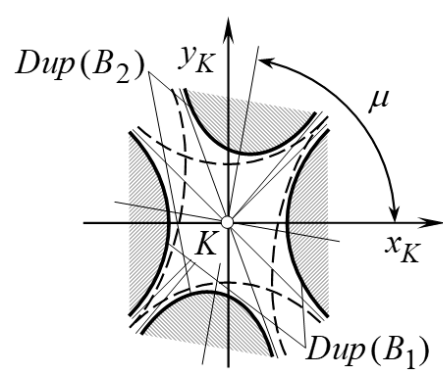

Minimal-to-hyperbolic (pseudo-concave)

10.9

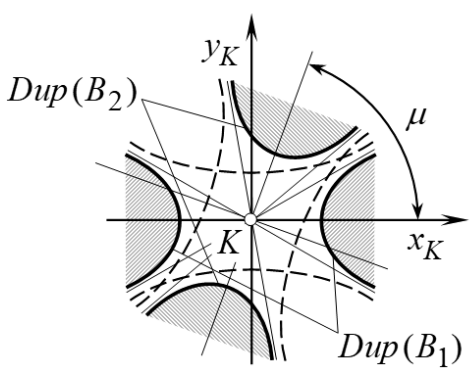

Hyperbolic-to-hyperbolic (pseudo-concave, bouth)

10.10

Fig. 12. Relative orientation of Dupin indicatrices, $\operatorname{Dup}\left(B_{1}\right)$ and $\operatorname{Dup}\left(B_{2}\right)$, at contact point of truepoint-contact kinematic pairs with hyperbolic-to-hyperbolic contact of the functional surfaces $B_{1}$ and $B_{2}$.

Sixth, there are a few different kinds of point-contact kinematic pairs composed of different kinds of elliptic, umbilic, and parabolic, functional surfaces, $B_{1}$ and $B_{2}$. In Fig. 7, kinematic pairs of this 


\section{www.vntr.ru}

№ 161, 2021

kind correspond to 3.1, 3.2, 4.1, 4.2, 7.1, and 7.2, contacts of the functional surfaces $B_{1}$ and $B_{2}$ (see Fig. 13):

- Convex elliptic-to-elliptic (concave)-contact kinematic pair

- Convex umbilic-to-elliptic (concave)-contact kinematic pair

- Convex elliptic-to-umbilic (concave)-contact kinematic pair

- Convex umbilic-to-umbilic (concave)-contact kinematic pair

- Convex elliptic-to-parabolic (concave)-contact kinematic pair

- Convex umbilic-to-parabolic (concave)-contact kinematic pair

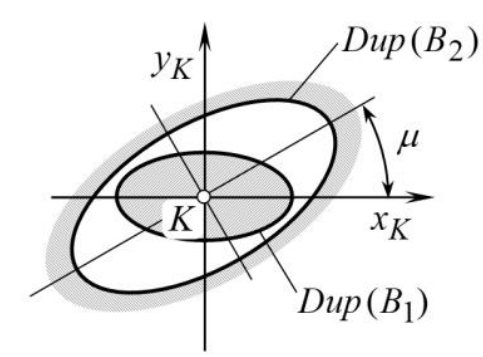

Elliptic-to-elliptic

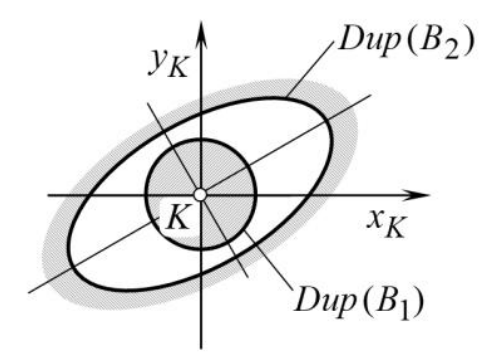

Umbilic-to-elliptic

Fig. 13. Relative orientation of the Dupin indicatrices, $\operatorname{Dup}\left(B_{1}\right)$ and $\operatorname{Dup}\left(B_{2}\right)$, at contact point of true-point-contact kinematic pairs with the functional surfaces $B_{1}$ and $B_{2}$ of elliptic, umbilic, and parabolic geometry.

For the kinematic pairs with contact of the kinds 3.1 and 7.1, the actual value of the angle, $\mu$, of the functional surfaces, $B_{1}$ and $B_{1}$, local relative orientation is in the range of: $\mu_{\min } \leq \mu \leq \mu_{\max }$.

The shown in Fig. 13 diagrams 3.1, 3.2, 4.1, and 4.2, correspond to the so-called conformal kinematic pairs. The term conformal kinematic pair is due to in all sections of the functional surfaces by a plane through the common perpendicular, normal curvature the lines of intersection of the functional surfaces are always of opposite sign. To a certain extent, the diagrams labeled 7.1 and 7.2 can also be considered as conformal kinematic pairs.

6.1.2. Locally-line-contact kinematic pairs. The locally-line-contact kinematic pairs (or just $L L_{c}-$ kinematic pairs, for simplicity) are those that feature zero difference between the magnitudes of radii of normal curvature, $R_{B .1}$ and $R_{B .2}$, in a section by a plane through a common perpendicular at contact point. A zero difference between the magnitudes of normal radii of curvature of two functional surfaces, $B_{1}$ and $B_{2}$, is observed when the radii of normal curvature are of equal magnitudes, and of opposite sign, that is, when the equality $R_{B .1}=-R_{B .2}$ is valid. Due to this feature, the minimal diameter, $d_{c n f}^{\min }$, of the indicatrix of conformity, $C_{n f}\left(B_{1} / B_{2}\right)$, at point of contact of the functional surfaces, $B_{1}$ and $B_{2}$, equals to zero, and, therefore, the equality $d_{c n f}^{\min } \equiv 0$ is always observed in all locally-extremal point-contact kinematic pairs. This is the reason for kinematic pairs of the kind under consideration are referred to as locally-extremal point-contact kinematic 
pairs [as they feature an extremal value of minimal diameter of the indicatrix of conformity ( $\left.\left.d_{c n f}^{\min } \equiv 0\right)\right]$.

Evidently, the locally-extremal point-contact kinematic pairs can be composed either by a convex and a concave local patches of the functional surfaces, $B_{1}$ and $B_{2}$, or by a convex and a saddle-like of the functional surfaces, $B_{1}$ and $B_{2}$. No locally-extremal kinematic pairs can be composed if both of the functional surfaces are concave, or one of them is concave, while another one is a saddle-like local patch of the functional surface.

Different kinds of kinematic pairs with locally line-contact of functional surfaces are distinguished. First, several different kinds of locally-line-contact kinematic pairs that are composed by either convex elliptic, or convex umbilic functional surfaces that make contact with concave functional surfaces of various geometries. The kinematic pairs of this kind are composed of convex and concave functional surfaces, $B_{1}$ and $B_{2}$ (see Fig. 14):

- Elliptic (concave)-to-elliptic (convex)-contact kinematic pair

- Elliptic (concave)-to-umbilic (convex)-contact kinematic pair

- Umbilic (concave)-to-elliptic (convex)-contact kinematic pair

- Parabolic (concave)-to-elliptic (convex)-contact kinematic pair

- Parabolic (concave)-to-umbilic (convex)-contact kinematic pair

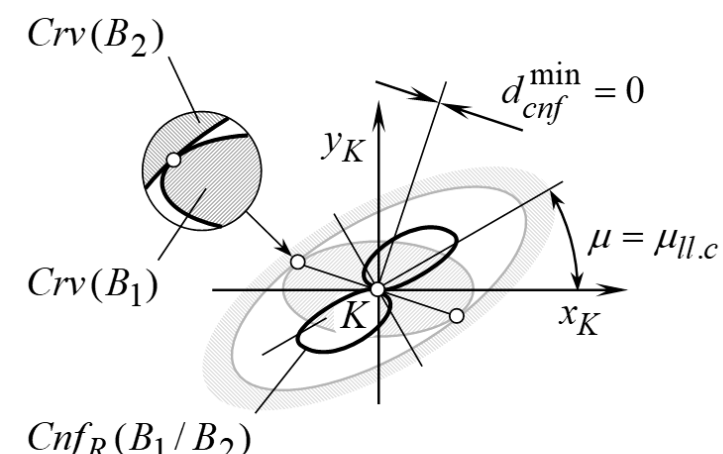

$\operatorname{Cnf}_{R}\left(B_{1} / B_{2}\right)$

Elliptic-to-elliptic

3.1

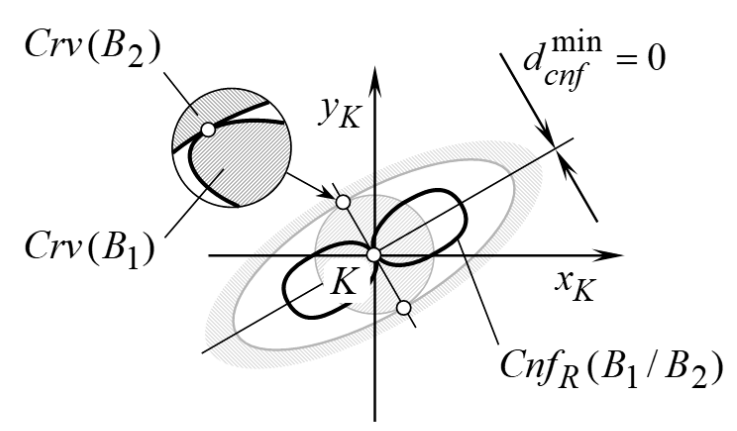

Umbilic-to-elliptic

3.2

Fig. 14. Indicatrices of conformity, $C n f_{R}\left(B_{1} / B_{2}\right)$, at contact point of locally-line-contact kinematic pairs with convex-to-concave contact of functional surfaces $B_{1}$ and $B_{1}$. 
www.vntr.ru

№ 161, 2021

Kinematic pairs of this kind feature a single contact point between the functional surfaces, $B_{1}$ and $B_{2}$, along radii of normal curvature of equal magnitude and opposite sign. In Fig. 7, kinematic pairs of this kind correspond to 3.1, 3.2, 4.1, 7.1, and 7.2, contacts of the functional surfaces $B_{1}$ and $B_{2}$. Indicatrices of conformity, $\operatorname{Cnf}_{R}\left(B_{1} / B_{2}\right)$, at contact point of the functional surfaces, $B_{1}$ and $B_{2}$ , of the kinematic pairs of this particular kind are shown in Fig. 14. There are no constraints onto the actual value of the angle of local relative orientation, $\mu$, of the functional surfaces, $B_{1}$ and $B_{2}$, for the kinematic pairs labeled 3.2, 4.1, and 7.2. For the kinematic pairs with contact of the kinds 3.1, and 7.1, the actual value of the angle, $\mu$, of the functional surfaces, $B_{1}$ and $B_{2}$, local relative orientation equals to a value $\mu=\mu_{l l . c}$ at which the contacting surfaces form a locally-line contact.

Second, numerous different kinds of locally-line point-contact kinematic pairs composed either of elliptic, or umbilic, or parabolic, functional surfaces that make contact with functional surfaces of hyperbolic geometry. Kinematic pairs of this kind feature a single contact point between the functional surfaces. In Fig. 7, kinematic pairs of this kind correspond to 8.1, 8.2, 8.5, 9.1, 9.2, 9.5, 10.1, 10.2, and 10.5, contacts of the functional surfaces $B_{1}$ and $B_{2}$ (see Fig. 15):

- Hyperbolic (pseudo-concave)-to-elliptic (convex)-contact kinematic pair

- Hyperbolic (minimal)-to-elliptic (convex)-contact kinematic pair

- Hyperbolic(pseudo-convex)-to-elliptic (convex)-contact kinematic pair

- Hyperbolic (pseudo-concave)-to-umbilic (convex)-contact kinematic pair

- Hyperbolic (minimal)-to-umbilic (convex)-contact kinematic pair

- Hyperbolic(pseudo-convex)-to-umbilic (convex)-contact kinematic pair

- Hyperbolic (pseudo-concave)-to-parabolic (convex)-contact kinematic pair

- Hyperbolic (minimal)-to-parabolic (convex)-contact kinematic pair

- Hyperbolic(pseudo-convex)-to-parabolic (convex)-contact kinematic pair

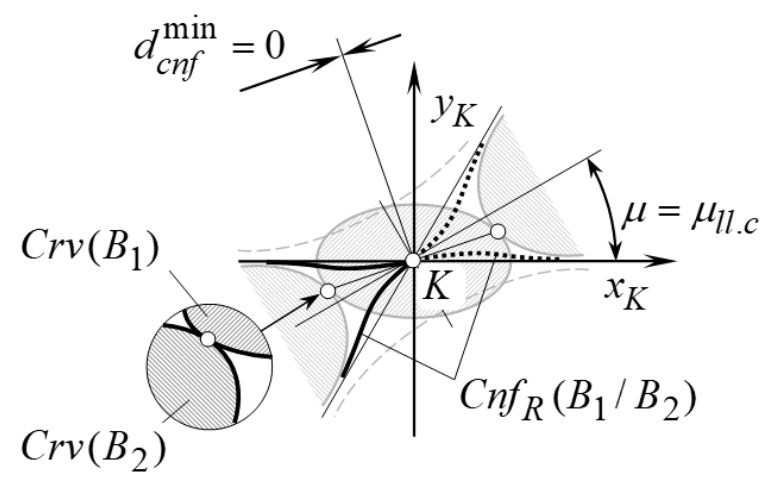

Elliptic-to-hyperbolic (pseudo-concave)

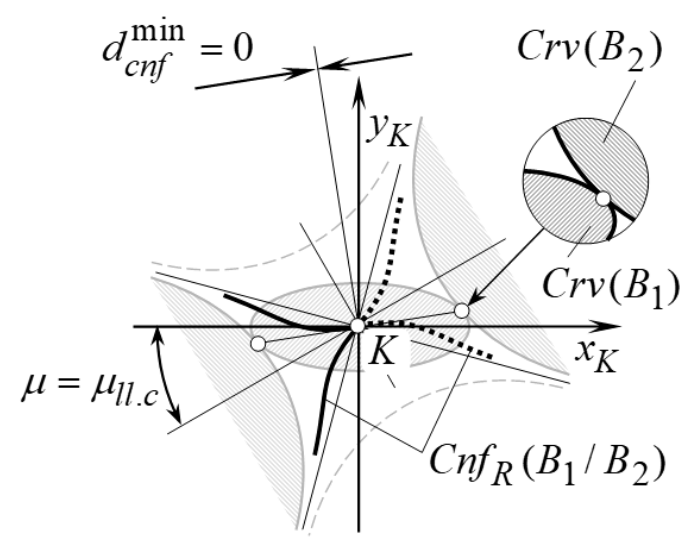

Elliptic-to-minimal (pseudo-umbilic)

\section{1}

Fig. 15. Indicatrices of conformity, $\operatorname{Cnf}_{R}\left(B_{1} / B_{2}\right)$, at contact point of locally-line kinematic pairs that feature hyperbolic-to-convex point-contact of the functional surfaces $B_{1}$ and $B_{1}$.

Two examples (8.1 and 9.1) of indicatrices of conformity, $C n f_{R}\left(B_{1} / B_{2}\right)$, at contact point of the functional surfaces, $B_{1}$ and $B_{2}$, of the kinematic pairs of this particular kind are shown in 15_Fig. 3.16. For the kinematic pairs with contact of the kinds 8.1, 8.5, 9.1, 9.5, 10.1, and 10.5, the 
actual value of the angle, $\mu$, of the functional surfaces local relative orientation equals to a value $\mu=\mu_{\text {ll.c }}$ at which the contacting surfaces form a locally-line contact. No constraints on the actual value of the angle, $\mu$, are imposed in the kinematic pairs with 8.2, 9.2, and 10.2 kinds of contact.

Third, there are three different kinds of locally-line point-contact kinematic pairs composed of hyperbolic functional surfaces that make contact with functional surfaces of hyperbolic geometry. Kinematic pairs of this particular kind feature a single contact point between the functional surfaces. In Fig. 7, kinematic pairs of this kind correspond to 10.8, 10.9, and 10.10, contacts of the functional surfaces $B_{1}$ and $B_{2}$ (see Fig. 16):

- Hyperbolic (pseudo-concave)-to-hyperbolic (pseudo-convex)-contact kinematic pair

- Hyperbolic (pseudo-concave)-to-hyperbolic (minimal)-contact kinematic pair

- Hyperbolic (pseudo-concave)-to-hyperbolic (pseudo-concave)-contact kinematic pair

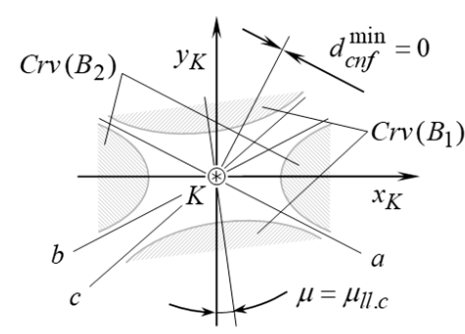

Hyperbolic (pseudo-concave)-to hyperbolic-(pseudo-convex)

10.8

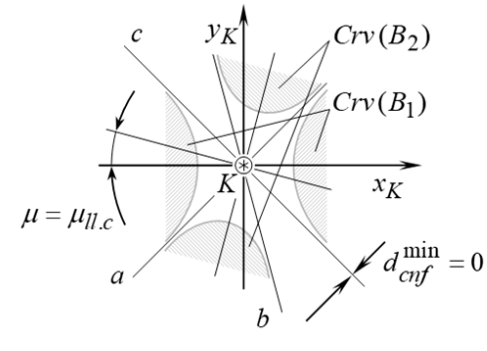

Minimal-to-hyperbolic (pseudo-convex)

10.9

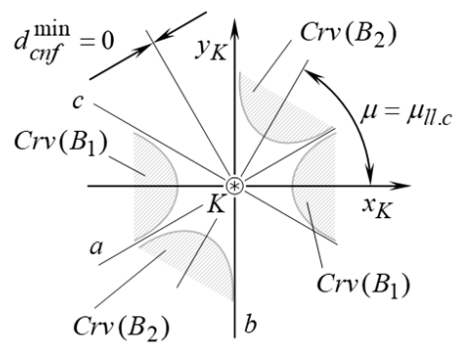

Hyperbolic (pseudo-concave)-to hyperbolic-(pseudo-concave)

10.10

Fig. 16. Relative orientation of curvature indicatrices, $\operatorname{Crv}\left(B_{1}\right)$ and $\operatorname{Crv}\left(B_{2}\right)$, at contact point of locally-line-contact kinematic pairs with hyperbolic-to-hyperbolic contact of the functional surfaces $B_{1}$ and $B_{1}$.

Relative orientation of the curvature indicatrices, $\operatorname{Crv}\left(B_{1}\right)$ and $\operatorname{Crv}\left(B_{2}\right)$, at contact point of kinematic pairs that feature hyperbolic-to-hyperbolic locally-line-contact of the functional surfaces, $B_{1}$ and $B_{1}$, are shown in Fig. 16. For the kinematic pairs of this design the actual value of the angle, $\mu$, of the functional surfaces local relative orientation equals to a value $\mu=\mu_{l l . c}$ at which the contacting surfaces form a locally-line contact.

6.1.3. Locally-surface-to-surface contact kinematic pairs I. The locally-surface-to-surface_I-contact kinematic pairs (or just $L S S_{c .1}$-kinematic pairs, for simplicity) are those featuring zero difference between magnitudes of the radii of normal curvature, $R_{B .1}$ and $R_{B .2}$, in all sections by a plane through common perpendicular at contact point of the functional surfaces. A zero difference between the magnitudes of normal radii of curvature of two functional surfaces, $B_{1}$ and $B_{2}$ is observed when the radii of normal curvature are of equal magnitude, and are of different sign, that is, when the equality $R_{B .1}=-R_{B .2}$ is valid. As this difference is extremely small (it is equal to zero), kinematic pairs of this kind are also referred to as locally-extremal-contact kinematic pairs.

Locally-surface-to-surface_I-contact kinematic pairs cannot be composed of two convex functional surfaces. Composed of convex functional surfaces, $B_{1}$ and $B_{2}$, kinematic pairs are not of the locally-surface-to-surface_I-contact kind.

Different kinds of kinematic pairs with locally-surface-to-surface_I-contact of functional surfaces are distinguished. 
www.vntr.ru

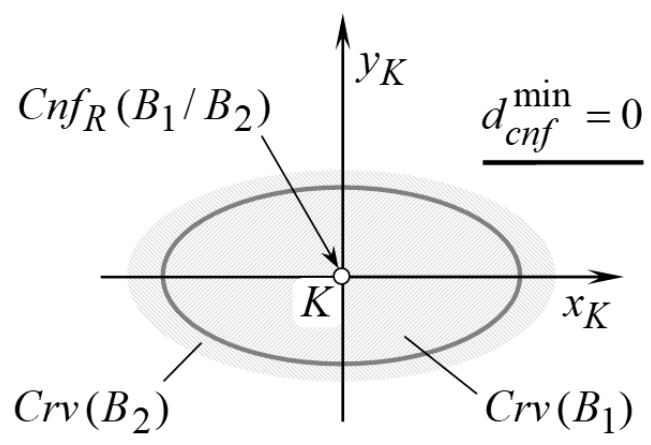

Elliptic (convex)-to-elliptic (concave)
№ 161, 2021

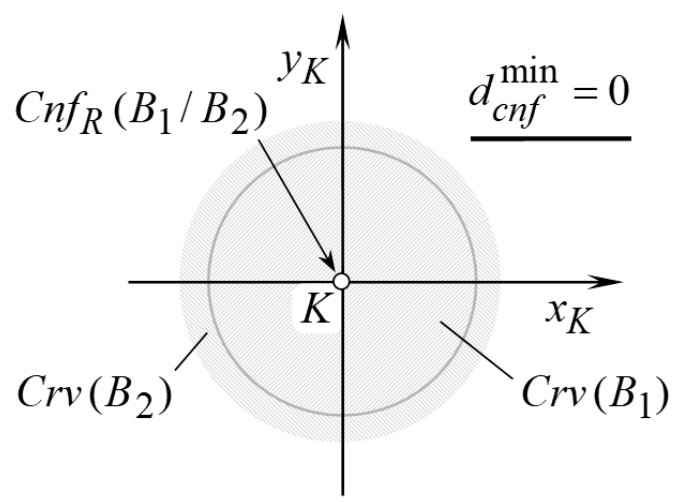

Umbilic (convex)-to-umbilic (concave)

\section{1}

4.2

Fig. 17. Indicatrices of conformity, $\operatorname{Cnf}_{R}\left(B_{1} / B_{2}\right)$, and relative orientation of the curvature indicatrices, $\operatorname{Crv}\left(B_{1}\right)$ and $\operatorname{Crv}\left(B_{2}\right)$, at contact point of point-contact kinematic pairs with hyperbolic-to-hyperbolic contact of functional surfaces $B_{1}$ and $B_{1}$. In all the kinematic pairs indicatrix of conformity, $\operatorname{Cnf}_{R}\left(B_{1} / B_{2}\right)$, is shrunk to a point coincident with contact point, $K$.

Locally-surface-to-surface_I-contact kinematic pairs of four different kinds composed by hyperbolic functional surfaces, $B_{1}$, that make contact with hyperbolic functional surfaces, $B_{2}$, of various geometries. The kinematic pairs of this kind are composed of two hyperbolic functional surfaces, $B_{1}$ and $B_{2}$ (see Fig. 17):

- Elliptic (concave)-to-elliptic (convex)-contact kinematic pair

- Umbilic (concave)-to-umbilic (convex)-contact kinematic pair

- Hyperbolic (concave)-to-hyperbolic (convex)-contact kinematic pair

- Hyperbolic (minimal)-to-hyperbolic (minimal)-contact kinematic pair

One more kinematic pair of the kind under consideration is represented with:

- Planar-to-planar-contact kinematic pair

Kinematic pairs of this kind feature single contact point between the functional surfaces, $B_{1}$ and $B_{2}$

- Radii of normal curvature in sections by a plane through the common perpendicular are of equal magnitude and of opposite sign. In Fig. 7, kinematic pairs of this kind correspond to 3.1, 4.2, 9.9, and 10.8, contacts of the functional surfaces $B_{1}$ and $B_{2}$. One can imagine that the principal radii of curvature, $R_{1_{B 1}}, R_{2_{B 1}}, R_{1_{B 2}}$, and $R_{2_{B 2}}$, in the kinematic pairs 3.1, 4.2, 9.9, and 10.8, approach an infinity $\left(R_{1_{B 1}} \rightarrow \infty, R_{2_{B 1}} \rightarrow \infty, R_{1_{B 2}} \rightarrow \infty\right.$, and $\left.R_{2_{B 2}} \rightarrow \infty\right)$. In such a scenario, the functional surfaces, $B_{1}$ and $B_{2}$, get flatten. Actually, flattening of the functional surfaces is observed in any and all kind of kinematic pairs, and not only in the designs 3.1, 4.2, 9.9, and 10.8. Therefore, in Fig. 17, locally-surface-to-surface_I-contact kinematic pairs with flatten functional surfaces, $B_{1}$ and $B_{2}$, are labeled as 1.1, 2.1, 2.2, 3.1, .., 10.9, and 10.10.

Relative orientation of the Dupin indicatrices, $\operatorname{Dup}\left(B_{1}\right)$ and $\operatorname{Dup}\left(B_{2}\right)$, at contact point of the kinematic pairs of this particular kind are shown in Fig. 17. For the kinematic pair labeled 3.1, 9.9, and 10.8, the actual value of the angle, $\mu$, of the functional surfaces, $B_{1}$ and $B_{2}$, local relative orientation equals to a value $\mu= \pm 90^{\circ}$ in all the locally-surface-to-surface_I-contact kinematic pairs. No constraints onto the actual value of the angle, $\mu$, of the functional surfaces, $B_{1}$ and $B_{2}$, local 
relative orientation is imposed in cases of hyperbolic (minimal)-to-hyperbolic (minimal)-contact kinematic pair (labeled 4.2).

The indicatrices of conformity, $\operatorname{Cnf}_{R}\left(B_{1} / B_{2}\right)$, in locally-surface-to-surface_I-contact kinematic pairs is always shrunk to a point. This point is coincident with the contact point, $K$, of the functional surfaces.

6.1.4. High-conformal point-contact kinematic pairs I. The power density of point-contact kinematic pairs is strongly correlated to the degree of conformity to one another of the functional surfaces, $B_{1}$ and $B_{2}$, at every point of their contact. The more the surfaces $B_{1}$ and $B_{2}$ are conformal at points of their contact, the higher the power density of the kinematic pair, and vice versa.

Qualitatively, the degree of conformity at a point of contact of two functional surfaces can be viewed as follows. Consider a section of two functional surfaces, $B_{1}$ and $B_{2}$, by a plane through the common perpendicular. The radii of normal curvature of the surfaces, $B_{1}$ and $B_{2}$, within the plane section are designated by $R_{B_{1}}$ and $R_{B_{2}}$, correspondingly. A mismatch of the radii of normal curvature of the surfaces, $R_{B_{1}}$ and $R_{B_{2}}$, is denoted by $\delta R$ :

$$
\delta R=\left|R_{B_{1}}-R_{B_{2}}\right|
$$

The functional surfaces, $B_{1}$ and $B_{2}$, are referred to as high-conformal point-contact kinematic pairs I (or just $H C_{c .1}$-kinematic pairs, for simplicity), if the actual value of the normal radii mismatch, $\delta R$, is in the range of:

$$
0<\delta R \leq[\delta R]
$$

where $[\delta R]$ is the maximum permissible mismatch of the radii of normal curvature of the surfaces, $R_{B_{1}}$ and $R_{B_{2}}$, at which the contact is still referred to high-conformal (see [16] for details on conformity criterion in kinematic pairs).

6.1.4.1. Conformity criterion in kinematic pairs. In order to establish a criterion by means of which conformal contact in kinematic pairs is separated from their high-conformal contact, consider two functional surfaces, $B_{1}$ and $B_{2}$, that are intersected by a normal plane through the contact point, $K$. The plane is constructed so as to be perpendicular to the common tangential straight line, $\mathbf{t}_{C L}$.

The plane section of the first functional surface is labeled $B_{1}$. Within the differential vicinity of the contact point, radius of curvature of the curve $B_{1}$ is labeled $R_{B_{1}}$. The radius, $R_{B_{1}}$, is of a negative value $\left(R_{B_{1}}<0\right)$, as in this example the functional surface $B_{1}$ is considered concave.

Prior to the operating load is applied, the plane section of the second functional surface is labeled $B_{2}^{*}$. After the load is applied and the second functional surface is slightly penetrated into the first functional surface, the same section, $B_{2}^{*}$, is labeled $B_{2}$. It is assumed here that within the differential vicinity of the point of contact, the radii of curvature of the curves of intersection of the surfaces, $B_{2}^{*}$ and $B_{2}$, are of the same value, $R_{B_{2}}$. The radius of curvature is of a positive value ( $R_{B_{2}}>0$ ), as in this example the second functional surface is considered convex.

The width, $l$, of the contact between the functional surfaces under an applied load can be expressed in terms of the radii of normal curvature, $R_{B_{1}}$ and $R_{B_{2}}[16]$ :

$$
l=l(\hat{k}, \hat{K})
$$




\section{Bulletin of Science and Technical Development}

\section{www.vntr.ru}

№ 161, 2021

In Eq. (8), all the design parameters are normalized by the functional surface, $B_{2}$, radius $R_{B_{2}}$. The normalized design parameters are as follows: $\hat{K}=R_{B_{1}} / R_{B_{2}}$, and $\hat{k}=k R_{B_{2}} / R_{B_{2}}$ [16].

The function $l=l(\hat{k}, \hat{K})$ is valid for all kinds of contact of the functional surfaces, $B_{1}$ and $B_{2}$. For conformal kinematic pairs, only cases of convex-to-concave contacts of the functional surfaces are of interest.

In Fig. 18, a three-dimensional plot of the function $l=l(\hat{k}, \hat{K})$ is constructed for the cases of convex-to-concave contacts of the functional surfaces, $B_{1}$ and $B_{2}$, in a kinematic pair.

A performed analysis of the $3 D$-plot allows the following conclusions.

The sections of the surface $l=l(\hat{k}, \hat{K})$ by the planes $\hat{k}_{i}=$ Const (see Fig. 18) are represented by the curves that have asymptotes. For a particular curve, $k_{i}=$ Const, shown in Fig. 18 in the bold line, the axis $l$ and the straight line $l=1$ are the asymptotes.

The greatest possible degree of mismatch in the curvature of the functional surfaces, $B_{1}$ and $B_{2}$, corresponds to an infinite value of the parameter $\hat{K} \rightarrow-\infty$.

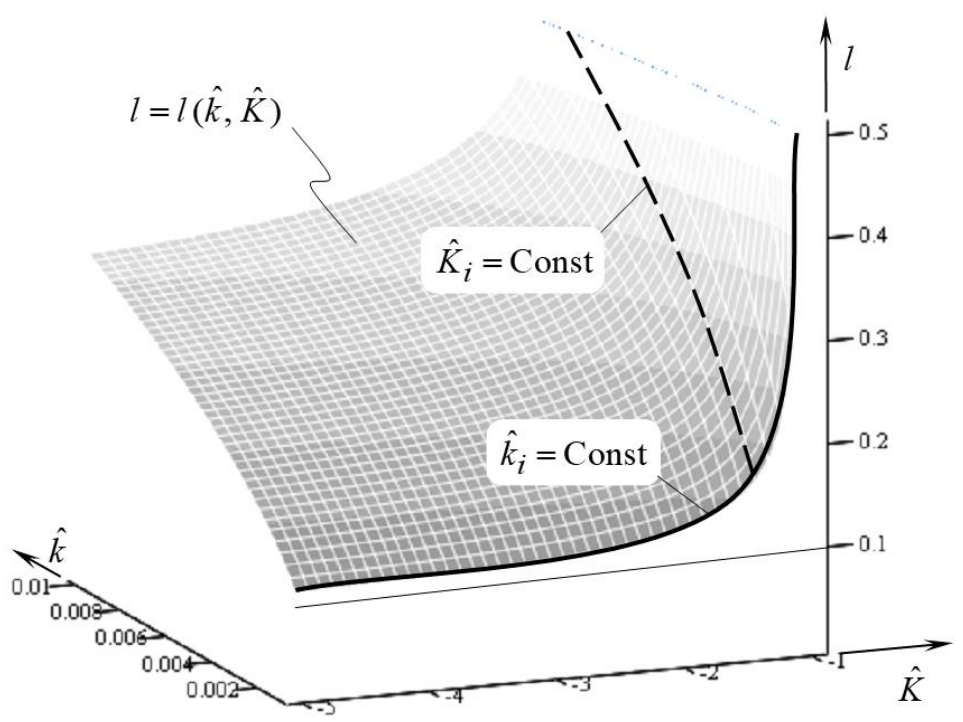

Fig. 18. Three-dimensional plot of function $l=l(\hat{k}, \hat{K})$ constructed An interval of alteration of the parameter $\hat{K}$ starting from $-\infty$ and going up to approximately $\hat{K}=-2$ can conveniently accommodate any desirable displacement of the functional surfaces, $B_{1}$ and $B_{2}$, from their correct location. However, in the range $-\infty<\hat{K}<-2$, an increase in the degree of conformity of the functional surfaces, $B_{1}$ and $B_{2}$, is negligibly small. In the range of variation of the parameter $\hat{K}$, the load-carrying capacity of a conformal kinematic pair remains approximately at the same value. Therefore, use of just the convex-to-concave contacts of the functional surfaces gives almost no improvement to the load-carrying capacity of kinematic pairs. For the convex-toconcave contacts, an additional requirement has to be fulfilled in order to significantly improve the load-carrying capacity of a conformal kinematic pair.

On the other hand, even a small alteration in the actual value of the parameter $\hat{K}$ within the interval $-2<\hat{K}<-1$ results in a significant increase in the degree of conformity of the functional surfaces, $B_{1}$ and $B_{2}$. This immediately entails a corresponding increase in the load-carrying capacity of the kinematic pair.

High-conformal kinematic pairs feature convex-to-concave contacts of the functional surfaces. Moreover, the degree of conformity at point of contact of the functional surfaces in high-conformal kinematic pairs exceeds a certain critical value (the threshold, in other words).

In the aforementioned example (see Fig. 18), the value of parameter $\hat{K}$ (that is, the value of $\hat{K} \approx-2$ ) can be referred to as a critical value, that is, $\hat{K}_{c r}$. This allows one to distinguish between conformal kinematic pairs (for which an inequality, $-\infty<\hat{K}<\hat{K}_{c r}$, is valid), and high-conformal kinematic pairs (for which an inequality, $\hat{K}_{c r} \leq \hat{K}<-1$, is valid). Because of the favorable conditions of contact of 
the functional surfaces, high-conformal kinematic pairs allow for a significantly greater power density.

Without going into the details of this analysis, it is clear that high-conformal kinematic pairs require tighter tolerances for any possible displacements of the functional surfaces, $B_{1}$ and $B_{2}$, from their desirable locations and orientations. This relates not just for the tolerances on the manufacturing errors, but to any and all possible displacements caused by thermal extension, elastic deflection, and so forth. Otherwise, there could be no future for high-conformal transmission system.

The separation from one another areas of existence of conformal and high-conformal kinematic pairs are schematically illustrated in Fig. 19.

The performed analysis of the $3 D$ plot shown in Fig. 18 can be extended, although the extension is a bit aside of the main stream of the subject of this research.

Consider sections of the surface $l=l(\hat{k}, \hat{K}) \quad$ intersected by planes $\hat{K}_{i}=$ Const (see Fig. 18). An example of such sections is shown by the bold dashed line. For high-conformal kinematic pairs, the parameter $\hat{K}_{i}$ for these lines is in the range of $\hat{K}_{c r} \leq \hat{K}_{i}<-1$. The degree of mismatch in the curvature of the functional surfaces in high-conformal kinematic pairs is smaller compared to that in conformal kinematic pairs.

The indicatrix of conformity $\operatorname{Cnf}_{R}\left(B_{1} / B_{2}\right) \quad$ [see Eq. (2)] [and

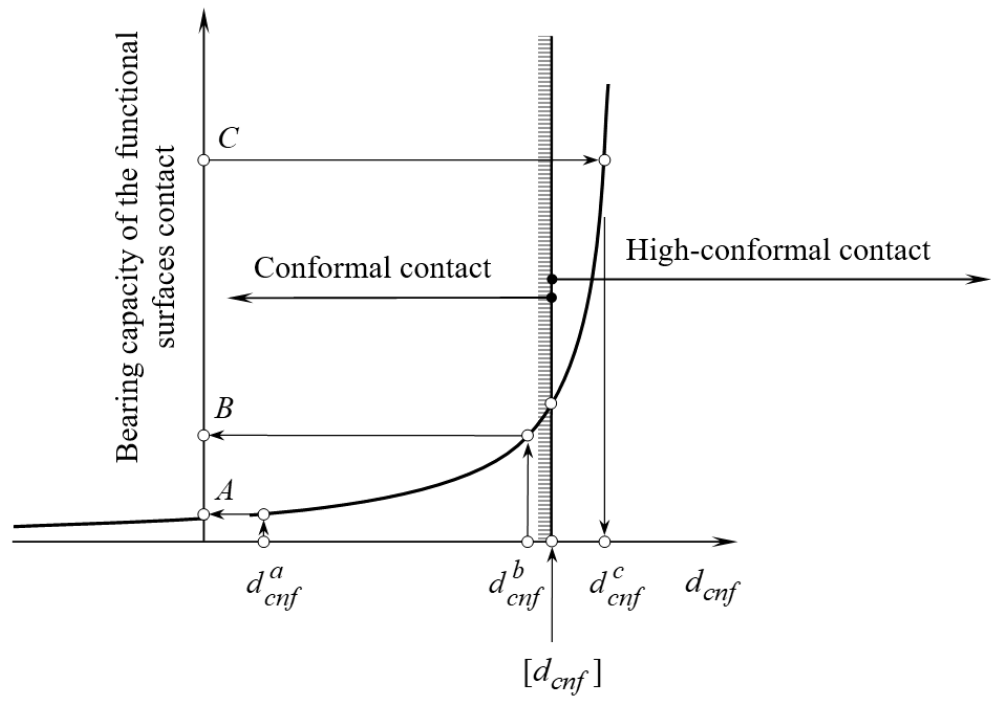

Fig. 19. Impact of degree of conformity, $d_{c n f}$, at contact point of functional surfaces, $B_{1}$ and $B_{2}$, onto the bearing capacity of the kinematic pair. inverse indicatrix of conformity, $\operatorname{Cnf}_{k}\left(B_{1} / B_{2}\right)$, as well] is developed aiming the analytical description of the contact geometry of two interacting functional surfaces, $B_{1}$ and $B_{2}$, in a kinematic pair. The minimum diameter, $d_{c n f}$, of the indicatrix of conformity, $C n f_{R}\left(B_{1} / B_{2}\right)$, at contact point, $K$, of the functional surfaces, $B_{1}$ and $B_{2}$, can be used as a quantitative measure of the degree of conformity of the interacting bodies in a kinematic pair. In high-conformal kinematic pairs, the degree of conformity of the functional surfaces, $B_{1}$ and, $B_{2}$, exceeds a threshold beyond which a significant increase in the bearing capacity of the interacting functional surfaces is observed. Schematically, this property of high-conformal kinematic pairs is illustrated in Fig. 20. 


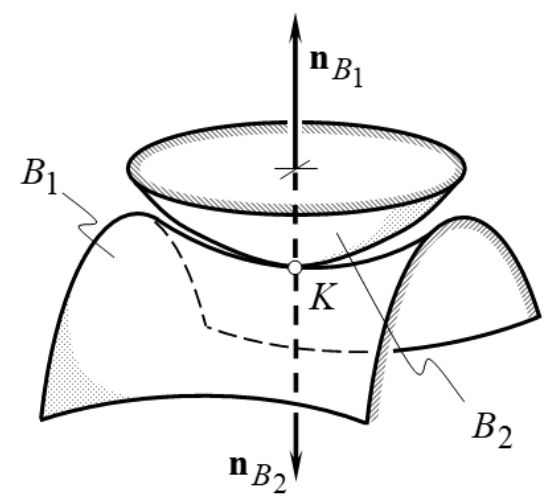

(a)

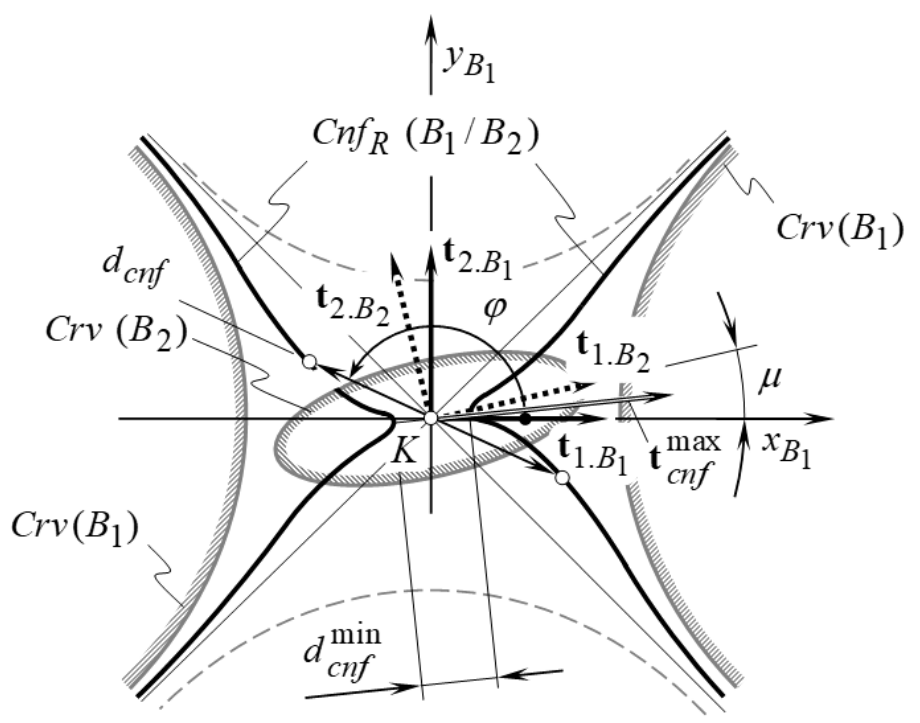

(b)

Fig. 20. An example of the indicatrix of conformity, $\operatorname{Cnf}_{R}\left(B_{1} / B_{2}\right)$, constructed at point of contact of the functional surfaces, $l$ and $B_{2}$, in a kinematic pair.

For a certain degree of conformity, $d_{c n f}^{a}$, at point of contact of functional surfaces, $B_{1}$ and $B_{2}$, the bearing capacity of the kinematic pair can be evaluated by a certain number, $A$. If degree of conformity of the functional surfaces, $B_{1}$ and $B_{2}$, is increased from $d_{c n f}^{a}$ to a value of $d_{c n f}^{b}$, an insignificant increase in the bearing capacity of the kinematic pair from a number $A$ to a number $B$ is observed. An increase in the bearing capacity is insignificant in the case under consideration as both the degrees of conformity, $d_{c n f}^{a}$ and $d_{c n f}^{b}$, are smaller than the threshold $\left[d_{c n f}\right]$, beyond which a significant increase in the bearing capacity of the functional surfaces, $B_{1}$ and $B_{2}$, occurs.

Let us assume that the degree of conformity, $d_{c n f}^{c}$, is greater than the threshold, $\left[d_{c n f}\right]$. When the inequality $d_{c n f}^{c}>\left[d_{c n f}\right]$ is valid, the bearing capacity of the functional surfaces, $B_{1} B_{2}$, grows much faster.

In high-conformal kinematic pairs, the inequality $d_{c n f}^{c} \geq\left[d_{c n f}\right]$ is always observed.

6.1.4.2. Kinds of high-conformal point-contact kinematic pairs I. At a single common point, $K$, high-conformal point-contact kinematic pairs feature convex-to-concave contact at least in a single plane through the common perpendicular (no high-conformal point-contact kinematic pairs I can be comprised by two convex functional surfaces, $B_{1}$ and $B_{2}$ ). Due to this feature, the minimal diameter, $d_{c n f}^{\min }$, of the indicatrix of conformity, $C n f_{R}\left(B_{1} / B_{2}\right)$, at point of contact of the functional surfaces, $B_{1}$ and $B_{2}$, exceeds zero, namely, the inequality: $d_{c n f}^{\min }>0$ is always observed in all kinds of high-conformal point-contact kinematic pairs. On top of that, in high-conformal point-contact kinematic pairs, the minimum diameter $d_{c n f}^{\min }$ of the indicatrix of conformity, $\operatorname{Cnf}_{R}\left(B_{1} / B_{2}\right)$, is in the range of $0<d_{c n f}^{\min } \leq\left[d_{c n f}^{\min }\right]$. Point-contact kinematic pairs of the kind under consideration are referred to as high-conformal, as they feature a very small difference between radii of normal 
curvature in a common section by a plane through a common perpendicular. The term "very small difference" can be quantified ${ }^{4}$.

First, there are two different kinds of high-conformal point-contact kinematic pairs I kinematic pairs that feature either elliptic, or umbilic, functional surface, $B_{2}$, in contact with a planar local patch of a mating functional surface, $B_{1}$ (see Fig. 21):

- Planar-to-elliptic-contact high-conformal kinematic pair

- Planar-to-umbilic-contact high-conformal kinematic pair

Kinematic pairs of this kind feature a single contact point between the functional surfaces $B_{1}$ and $B_{2}$. In Fig. 7, kinematic pairs of this kind correspond to $\mathbf{6 . 1}$, and $\mathbf{6 . 2}$, contacts of the functional surfaces $B_{1}$ and $B_{2}$. Details on kinematic pairs of this particular kind are shown in Fig. 21. There are no constraints onto the actual value of the angle of local relative orientation, $\mu$, of the functional surfaces, $B_{1}$ and $B_{2}$, for the kinematic pairs having contact of kinds 6.1, and 6.2.

Second, there are several different

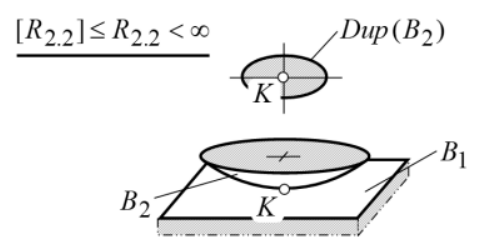

Convex elliptic-to-plane

6.1

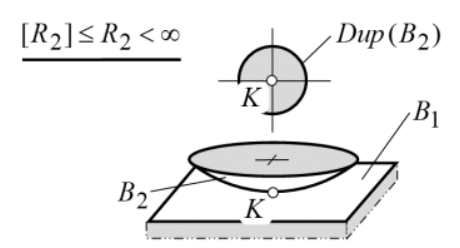

Convex umbilic-to-plane

6.2

Fig. 21. Details on high-conformal point-contact_I kinematic pairs that feature a planar functional surface $B_{1}$ in contact

with either elliptic, or umbilic functional surface $B_{1}$. kinds of convex-to-hyperbolic high-conformal point-contact kinematic pairs I that feature different kinds of hyperbolic functional surface in contact with convex local patches of a mating functional surface. Kinematic pairs of this kind feature a single contact point between the functional surfaces $B_{1}$ and $B_{2}$. In Fig. 7, kinematic pairs of this kind correspond to 8.1, 8.2, 9.1, 9.2, 10.1, and 10.2, contacts of the functional surfaces $B_{1}$ and $B_{2}$. The kinematic pairs of this kind are composed of convex functional surfaces, $B_{1}$ and $B_{2}$, of the following geometries (see Fig. 22):

- Elliptic-to-hyperbolic (pseudo-concave) high-conformal point-contact kinematic pairs I

- Umbilic-to-hyperbolic (pseudo-concave) high-conformal point-contact kinematic pairs I

- Elliptic-to-minimal (pseudo-umbilic) high-conformal point-contact kinematic pairs I

- Umbilic-to-minimal (pseudo-umbilic) high-conformal point-contact kinematic pairs I

- Elliptic-to-hyperbolic (pseudo-convex) high-conformal point-contact kinematic pairs I

- Umbilic-to-hyperbolic (pseudo-convex) high-conformal point-contact kinematic pairs I

The following condition, $0<d_{c n f}^{\min } \leq\left[d_{c n f}^{\min }\right]$, is valid for all six kinematic pairs listed above.

For the kinematic pairs with contact of kinds 8.1, 9.1, and 10.1, the actual value of the angle, $\mu$, of the functional surfaces local relative orientation is in the range of $\mu_{\min }<\mu<\mu_{\max }$, correspondingly.

\footnotetext{
${ }^{4}$ It is commonly adopted that high-conformal point-contact kinematic pairs I (as well, as high-conformal point-contact kinematic pairs II below) cannot be composed of two convex functional surfaces, $B_{1}$ and $B_{2}$, by a convex and a flatten surfaces, and so forth. This is correct to a certain extent. For example, one can imagine a convex functional surface, $B_{1}$, of elliptical kind with a flatten functional surface, $B_{2}$. If the principal radii of curvature, $R_{1_{B .1}}$ and $R_{2}$, of the functional surface, $B_{1}$, approach an infinity $\left(R_{1_{B .1}} \rightarrow \infty\right.$ and $R_{2} \rightarrow \infty$ ), then any desirable degree of conformity of two convex functional surfaces, $B_{1}$ and $B_{2}$, can be attained. Kinematic pairs of this particular kind (as well, as of similar kinds) are not discussed in detail in this research. The same is valid with respect to convex-to-convex contacts of the functional surfaces.
} 


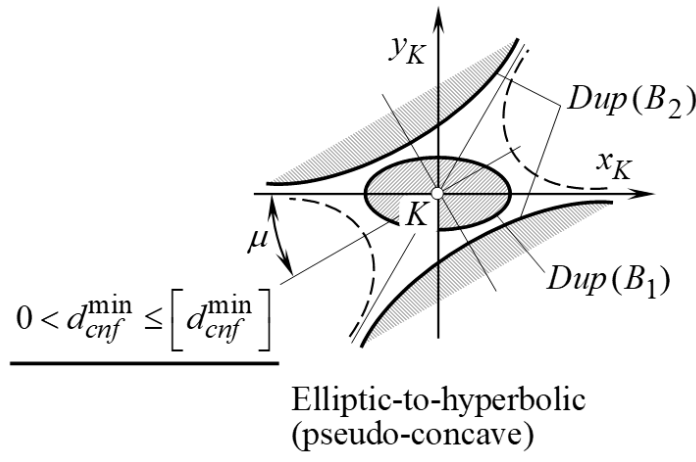

8.1

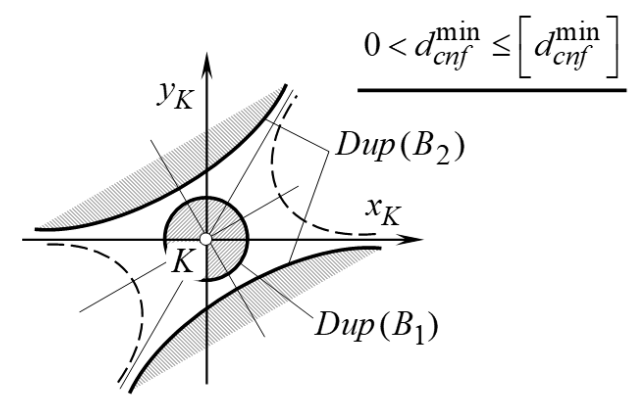

Umbilic-to-hyperbolic (pseudo-concave)

8.2

Fig. 22. Dupin indicatrices, $\operatorname{Dup}\left(B_{1}\right)$ and $\operatorname{Dup}\left(B_{2}\right)$, at contact point of high-conformal point-contact_I

kinematic pairs that feature convex-to-saddle-like point-contact of the functional surfaces $B_{1}$ and $B_{1}$.

There are no constraints onto the actual value of the angle of local relative orientation, $\mu$, of the functional surfaces, $B_{1}$ and $B_{2}$, for the kinematic pairs with contact of kinds 8.2, 9.2, and 10.2.

Third, there are three different kinds of convex-to-hyperbolic high-conformal point-contact kinematic pairs I that feature different kinds of hyperbolic functional surfaces in contact with convex parabolic local patches of a mating functional surface. Kinematic pairs of this kind feature a single contact point between the functional surfaces $B_{1}$ and $B_{2}$. In Fig. 7, kinematic pairs of this kind correspond to 8.5, 9.5, and 10.5, contacts of the functional surfaces $B_{1}$ and $B_{2}$ (see Fig. 23):

- Parabolic-to-hyperbolic (pseudo-concave)-contact kinematic pair

- Parabolic-to-minimal (pseudo-umbilic)-contact kinematic pair

- Parabolic-to-hyperbolic (pseudo-convex)-contact kinematic pair

For the kinematic pairs of these three kinds, the actual value of the angle, $\mu$, of the functional surfaces local relative orientation is in the range of $\mu_{\min }<\mu<\mu_{\max }$.

Fourth, there are three different kinds of hyperbolic-to-hyperbolic high-conformal point-contact

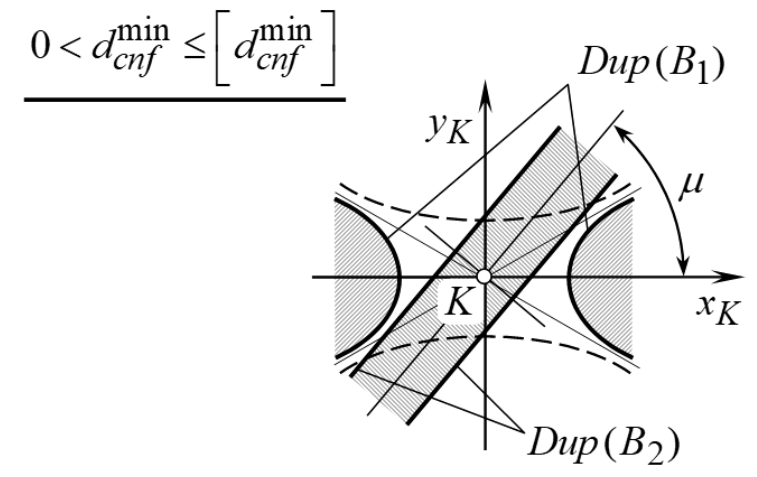

Parabolic-to-hyperbolic (pseudo-convex)

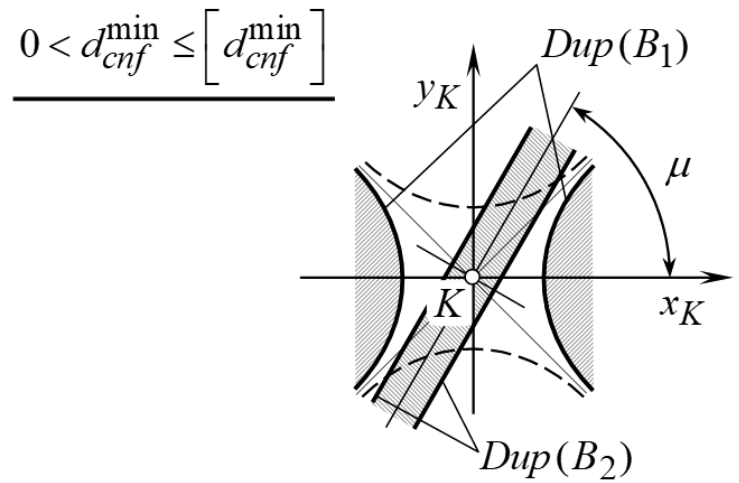

Parabolic-to-minimal (pseudo-umbilic)

\section{5}

9.5

Fig. 23. Relative orientation of Dupin indicatrices, $\operatorname{Dup}\left(B_{1}\right)$ and $\operatorname{Dup}\left(B_{2}\right)$, at contact point of high-conformal point-contact_I kinematic pair that features convex-parabolic-to-hyperbolic 
WwW.vntr.ru

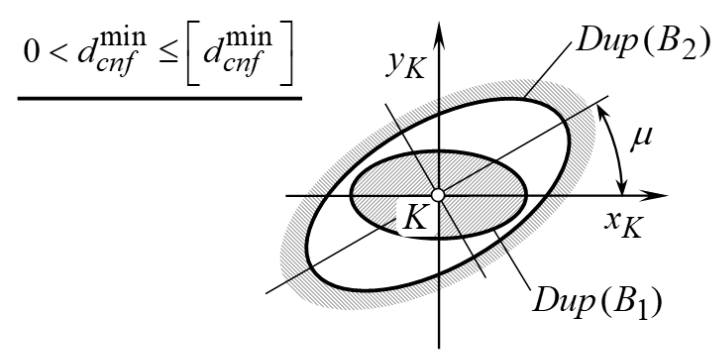

Elliptic-to-elliptic

№ 161, 2021 год

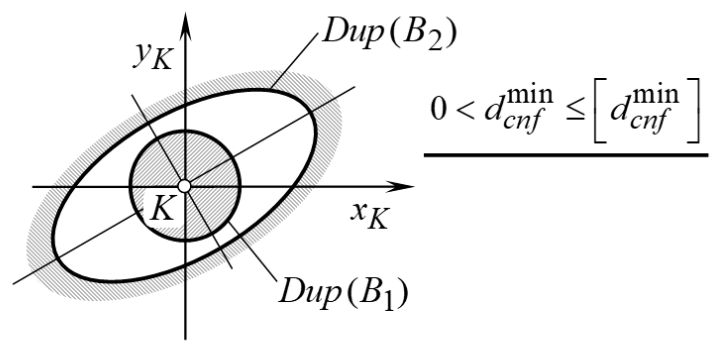

Umbilic-to-elliptic

3.2

Fig. 24. Relative orientation of Dupin indicatrices, $\operatorname{Dup}\left(B_{1}\right)$ and $\operatorname{Dup}\left(B_{2}\right)$, at contact point of true-point-contact kinematic pairs that feature concave elliptical functional surface $B_{1}$ in contact with convex surface $B_{1}$ of elliptic, and umbilic geometry.

kinematic pairs I that feature different kinds of hyperbolic functional surfaces in contact with hyperbolic local patches of a mating functional surface. Kinematic pairs of this kind feature a single contact point between the functional surfaces $B_{1}$ and $B_{2}$. In Fig. 7, kinematic pairs of this kind correspond to $\mathbf{1 0 . 8}, \mathbf{1 0 . 9}$, and 10.10, contacts of the functional surfaces $B_{1}$ and $B_{2}$ (see Fig. 24):

- Hyperbolic (pseudo-convex)-to-hyperbolic (pseudo-concave)-contact kinematic pair

- Hyperbolic (minimal)-to-hyperbolic (pseudo-concave)-contact kinematic pair

- Hyperbolic (pseudo-concave)-to-hyperbolic (pseudo-concave)-contact kinematic pair

For the kinematic pairs of these three kinds, the actual value of the angle, $\mu$, of the functional surfaces local relative orientation is in the range of $\mu_{\min }<\mu<\mu_{\max }$.

Fifth, there are a few different kinds of high-conformal point-contact kinematic pairs I composed of different kinds of elliptic, umbilic, and parabolic, functional surfaces in contact. Kinematic pairs of this kind feature a single contact point between the functional surfaces $B_{1}$ and $B_{2}$. In Fig. 7, kinematic pairs of this kind correspond to 3.1, 3.2, 4.1, 4.2, 7.1, and 7.2, contacts of the functional surfaces $B_{1}$ and $B_{2}$ (see Fig. 25):

- Elliptic (convex)-to-elliptic (concave)-contact kinematic pair

- Umbilic (convex)-to-elliptic (concave)-contact kinematic pair

- Elliptic (convex)-to-umbilic (concave)-contact kinematic pair

- Umbilic (convex)-to-umbilic (concave)-contact kinematic pair

- Elliptic (convex)-to-parabolic (concave)-contact kinematic pair

- Umbilic (convex)-to-parabolic (concave)-contact kinematic pair

For the kinematic pairs of these three kinds, the actual value of the angle, $\mu$, of the functional surfaces local relative orientation is in the range of $\mu_{\min }<\mu<\mu_{\max }$.

Summarizing, one can conclude that all possible kinds of point-contact kinematic pairs:

- $\quad$ are composed of two functional surfaces $B_{1}$ and $B_{2}$ having one point in common

- feature five degrees of freedom

- the minimal diameter, $d_{c n f}^{\min }$, of indicatrix of conformity, $C n f_{R}\left(B_{1} / B_{2}\right)$, at point of contact of the functional surfaces, $B_{1}$ and $B_{2}$, is always of a positive value, that is, the inequality: $d_{c n f}^{\min }>0$ is always valid in point-contact kinematic pairs

Point contact kinematic pairs of all kinds are covered in the discussion in section 6.1. 


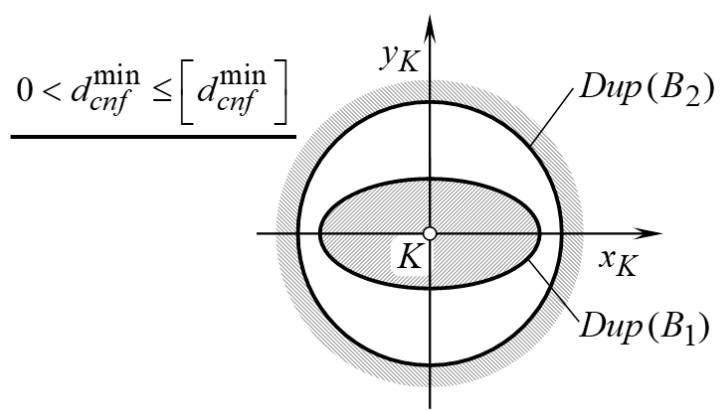

Elliptic-to-umbilic

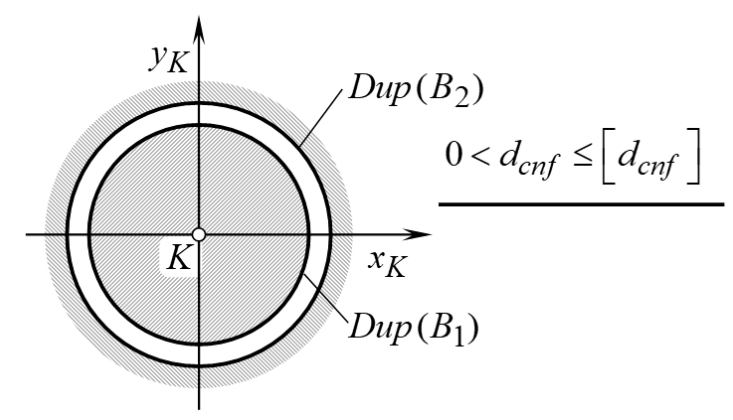

Umbilic-to-umbilic

4.1

Fig. 25. Relative orientation of Dupin indicatrices, Dup $\left(B_{1}\right)$ and $\operatorname{Dup}\left(B_{2}\right)$, at contact point of true-point-contact kinematic pairs that feature concave functional surfaces $B_{1}$ and $B_{1}$ of either elliptic, or umbilic, or parabolic type.

6.2. Line-contact kinematic pairs. In line-contact kinematic pairs (or just $L_{c}$-kinematic pairs, for simplicity), the functional surfaces, $B_{1}$ and $B_{2}$, make contact along an arc of a curve. A more in detail analysis reveals that the following kinds of contact between functional surfaces, $B_{1}$ and $B_{2}$, are recognized in line-contact kinematic pairs.

6.2.1. True-line-contact kinematic pairs. The true-line-contact kinematic pairs (or just $T L_{c}-$ kinematic pairs, for simplicity) of different kinds are distinguished. In true-line-contact kinematic pairs, the functional surfaces, $B_{1}$ and $B_{2}$, make contact along an arc of a curve. At every point of the line of contact, the arc of contact is entirely (locally) located in a section of the surfaces by a plane through the common perpendicular. In a particular case, this plane can be congruent to one of two principal planes of the surfaces. There is no correlation between radii of normal curvature in the rest of the normal planes. True-line-contact kinematic pairs of no design can be composed by two convex functional surfaces in contact.

First, there are numerous different kinds of true-line-contact kinematic pairs that are composed by either convex elliptic, or convex umbilic, or parabolic functional surfaces that make contact with concave functional surfaces of various geometries. The kinematic pairs of this kind are composed of two convex functional surfaces, $B_{1}$ and $B_{2}$ (see Fig. 7):

- $\quad$ Elliptic (concave)-to-elliptic (convex)-contact kinematic pair (3.1)

- Elliptic (concave)-to-umbilic (convex)-contact kinematic pair (3.2)

- Umbilic (concave)-to-elliptic (convex)-contact kinematic pair (4.1)

- Parabolic (convex)-to-parabolic (convex)-contact kinematic pair (5.5)

- Parabolic (convex)-to-parabolic (convex)-contact kinematic pair (6.5)

- Parabolic (concave)-to-flatten-contact kinematic pair (7.1)

- Parabolic (concave)-to-umbilic (convex)-contact kinematic pair (7.2)

- Parabolic (convex)-to-parabolic (convex)-contact kinematic pair (7.5) 
In Fig. 7, kinematic pairs of this kind correspond to 3.1, 3.2, 4.1, 5.5, 6.5, 7.1, 7.2, and 7.5, contacts of the functional surfaces $B_{1}$ and $B_{2}$. Indicatrices of conformity, $\operatorname{Cnf}_{R}\left(B_{1} / B_{2}\right)$, along with the Dupin indicatrices, $\operatorname{Dup}\left(B_{1}\right)$ and $\operatorname{Dup}\left(B_{2}\right)$, at contact point of the kinematic pairs of this particular kind are shown in Fig. 26. There are no constraints onto the actual value of the angle of local relative orientation, $\mu$, of the contacting functional surfaces, $B_{1}$ and $B_{2}$, for the kinematic pairs labeled 3.2, 4.1, 6.5, and 7.2. For the kinematic pair labeled 3.1, 5.5, 6.5, and 7.1, the actual value of the angle, $\mu$, of the functional surfaces, $B_{1}$ and $B_{2}$, local relative orientation equals to $\mu= \pm 180^{\circ}$.

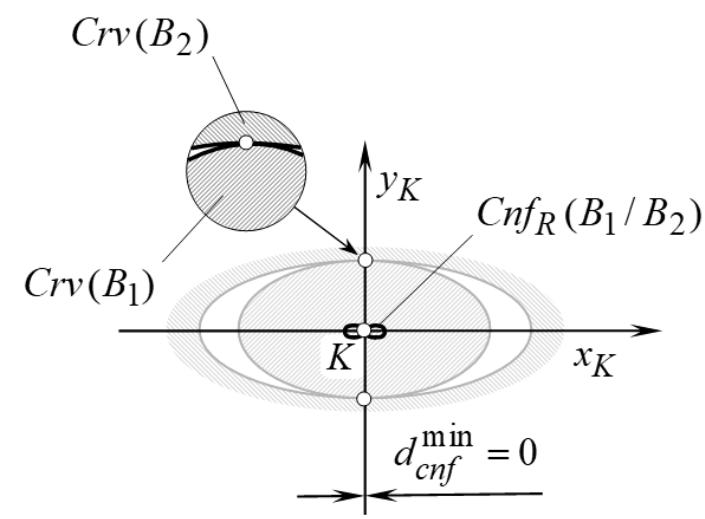

Elliptic-to-elliptic

3.1

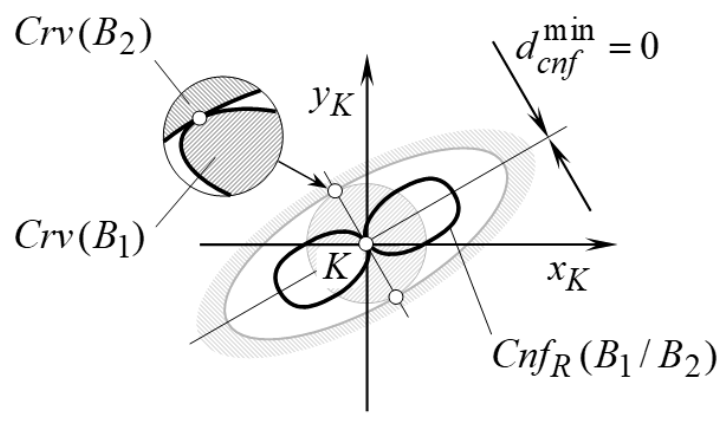

Umbilic-to-elliptic

3.2

Fig. 26. Indicatrices of conformity, $\operatorname{Cnf}_{R}\left(B_{1} / B_{2}\right)$, at contact point of true-line-contact kinematic pairs

Second, there are several different kinds of true-line-contact kinematic pairs composed of elliptic, umbilic, and parabolic, functional surfaces that make contact with functional surfaces of hyperbolic geometry. Kinematic pairs of this kind feature a single contact point between the functional surfaces. In Fig. 7, kinematic pairs of this kind correspond to 8.1, 8.2, 8.5, 9.1, 9.2, 9.5, 10.1, 10.2, and 10.5, contacts of the surfaces, $B_{1}$ and $B_{2}$ (see Fig. 27):

- Hyperbolic (pseudo-concave)-to-elliptic (convex)-contact kinematic pair (8.1)

- Hyperbolic (minimal)-to-elliptic (convex)-contact kinematic pair (8.2)

- Hyperbolic(pseudo-convex)-to-elliptic (convex)-contact kinematic pair (8.5)

- Hyperbolic (pseudo-concave)-to-umbilic (convex)-contact kinematic pair (9.1)

- Hyperbolic (minimal)-to-umbilic (convex)-contact kinematic pair (9.2)

- Hyperbolic(pseudo-convex)-to-umbilic (convex)-contact kinematic pair (9.5)

- Hyperbolic (pseudo-concave)-to-parabolic (convex)-contact kinematic pair (10.1)

- Hyperbolic (minimal)-to-parabolic (convex)-contact kinematic pair (10.2)

- Hyperbolic(pseudo-convex)-to-parabolic (convex)-contact kinematic pair (10.5)

For the kinematic pairs of the kinds labeled as 8.1, 8.2, 8.5, 10.1, 10.2, and 10.5, the actual value of the angle, $\mu$, of the functional surfaces local relative orientation equals to $\mu= \pm 180^{\circ}$. No constraints on the actual value of the angle, $\mu$, are imposed in the kinematic pairs $8.2,9.2,9.5$, and 10.2 . 


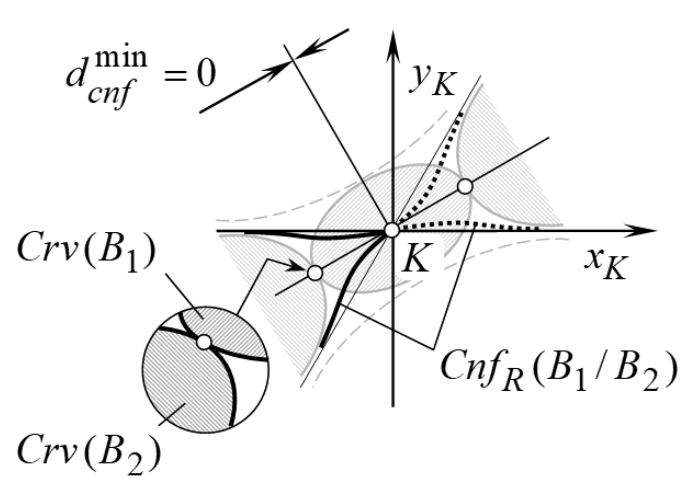

Elliptic-to-hyperbolic (pseudo-concave)

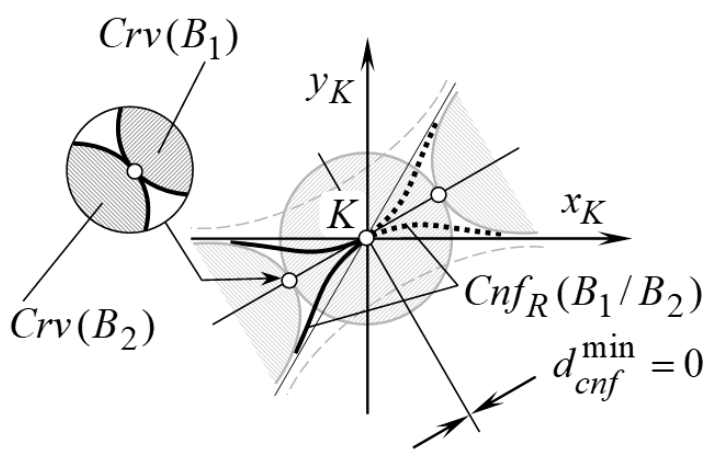

Umbilic-to-hyperbolic (pseudo-concave)

\section{1}

\section{2}

Fig. 27. Indicatrices of conformity, $\operatorname{Cnf}_{R}\left(B_{1} / B_{2}\right)$, at contact point of true-line-contact

kinematic pairs

6.2.2. Locally surface-to-surface contact kinematic pairs II. The locally-surface-to-surface_IIcontact kinematic pairs (or just $L_{S S_{c .2}}$-kinematic pairs, for simplicity) are those that feature true line contact of the functional surfaces in a section by a normal plane, and zero difference between magnitudes of radii of normal curvature, $R_{B .1}$ and $R_{B .2}$, in all the rest sections by a plane through a common perpendicular at contact point of the functional surfaces. Therefore, the Dupin indicatrices, $\operatorname{Dup}\left(B_{1}\right)$ and $\operatorname{Dup}\left(B_{2}\right)$, are congruent, and, thus, all the diameters of the indicatrix of conformity, $C n f_{R}\left(B_{1} / B_{2}\right)$, are of a zero value, $d_{c n f}^{\min }=0$.

Locally-surface-to-surface_II-contact kinematic pairs cannot be composed of two convex functional surfaces, $B_{1}$ and $B_{2}$.

Different kinds of kinematic pairs with locally-surface-to-surface_II-contact of functional surfaces are distinguished.

There are four different kinds of locally-surface-to-surface_II-contact kinematic pairs that are composed by functional surfaces, $B_{1}$ and $B_{2}$, of various geometries (see Fig. 28):

- Elliptic (convex)-to-elliptic (concave) locally-surface-to-surface_II-contact kinematic pair

- Parabolic (convex)-to-parabolic (concave) locally-surface-to-surface_II-contact kinematic pair

- Hyperbolic (minimal)-to-hyperbolic (minimal) locally-surface-to-surface_II-contact kinematic pair

- Hyperbolic (pseudo-concave)-to-hyperbolic (pseudo-convex) locally-surface-to-surface_IIcontact kinematic pair

Kinematic pairs of this kind feature a common line of contact point between the functional surfaces, $B_{1}$ and $B_{2}$, along with equal magnitude and of opposite sign radii of normal curvature in sections by a plane through the common perpendicular. In Fig. 7, kinematic pairs of this kind correspond to 
www.vntr.ru

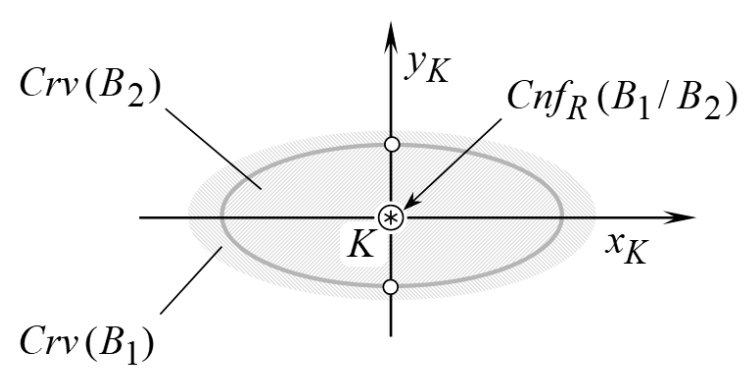

Elliptic (convex)-toelliptic (concave)

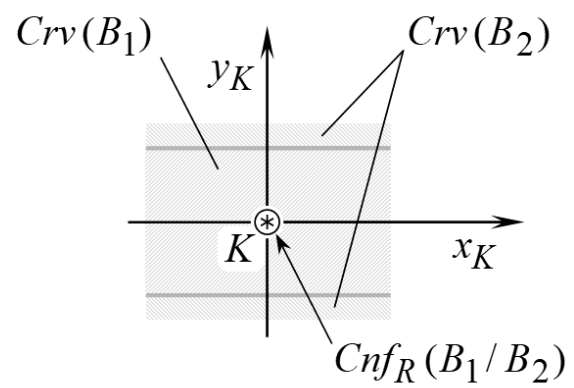

Parabolic (convex)-toparabolic (concave)

\section{5}

Fig. 28. Indicatrices of conformity, $\operatorname{Cnf}_{R}\left(B_{1} / B_{2}\right)$, and curvature indicatrices, $\operatorname{Crv}\left(B_{1}\right)$ and $\operatorname{Crv}\left(B_{2}\right)$, at contact point of locally-surface-to-surface_II-contact kinematic pairs.

3.1, 7.5, 9.9, and 10.8, contacts of the functional surfaces $B_{1}$ and $B_{2}$. One can imagine a situation that the radii of curvature, $R_{1_{B 1}}, R_{2_{B 1}}, R_{1_{B 2}}$, and $R_{2_{B 2}}$, in the kinematic pairs 3.1, 4.2,9.9, and 10.8, approach in infinity $\left(R_{1_{B 1}} \rightarrow \infty, R_{2_{B 1}} \rightarrow \infty, R_{1_{B 2}} \rightarrow \infty\right.$, and $\left.R_{2_{B 2}} \rightarrow \infty\right)$. In such a scenario, the functional surfaces, $B_{1}$ and $B_{2}$, are getting flatten. Actually, flattening of the functional surfaces is observed in any and all kind of kinematic pairs, and not only in the designs 3.1, 4.2, 9.9, and 10.8. Therefore, in Fig. 28, locally-surface-to-surface_I-contact kinematic pairs with flatten functional surfaces, $B_{1}$ and $B_{2}$, are labeled as 1.1, 2.1, 2.2, 3.1, .., 10.9, and 10.10.

Relative orientation of the curvature indicatrices, $\operatorname{Crv}\left(B_{1}\right)$ and $\operatorname{Crv}\left(B_{2}\right)$, at contact point of the kinematic pairs of this particular kind are shown in Fig. 28. For the kinematic pairs under consideration, the actual value of the angle, $\mu$, of the functional surfaces, $B_{1}$ and $B_{2}$, local relative orientation equals to a value $\mu= \pm 180^{\circ}$.

The indicatrices of conformity, $\operatorname{Cnf}_{R}\left(B_{1} / B_{2}\right)$, for kinematic pairs featuring locally-surface-tosurface_II-contact of the functional surfaces, $B_{1}$ and $B_{2}$, is always shrunk to a point. This point is coincident with the contact point of the functional surfaces.

6.2.3. High-conformal point-contact kinematic pairs II. The power density of kinematic pairs is strongly correlated to the degree of conformity of the functional surfaces, $B_{1}$ and $B_{2}$, to each other at every point of their contact. The more conformal the surfaces $B_{1}$ and $B_{2}$, the better power density of the kinematic pair, and vice versa.

The high-conformal point-contact kinematic pairs II (or just $H_{c .2}$-kinematic pairs, for simplicity) feature true line contact of the functional surfaces in one of two principal sections, and a small difference between magnitudes of radii of normal curvature, $R_{B .1}$ and $R_{B .2}$, in all the rest sections by a plane through a common perpendicular at contact point of the functional surfaces. Therefore, the curvature indicatrices, $\operatorname{Crv}\left(B_{1}\right)$ and $\operatorname{Crv}\left(B_{2}\right)$, are congruent, and, thus, all the diameters of the indicatrix of conformity, $\operatorname{Cnf}_{R}\left(B_{1} / B_{2}\right)$, are of a zero value, $d_{c n f}^{\min }=0$.

Four different kinds of high-conformal point-contact kinematic pairs II composed of functional surfaces, $B_{1}$ and $B_{2}$, of various geometries are distinguished (see Fig. 29):

- Elliptic (convex)-to-elliptic (concave) locally-surface-to-surface_II-contact kinematic pair

- Parabolic (convex)-to-parabolic (concave) locally-surface-to-surface_II-contact kinematic pair 
www.vntr.ru

№ 161, 2021

- Hyperbolic (minimal)-to-hyperbolic (minimal) locally-surface-to-surface_II-contact kinematic pair

- $\quad$ Hyperbolic (pseudo-concave)-to-hyperbolic (pseudo-convex) locally-surface-to-surface_IIcontact kinematic pair

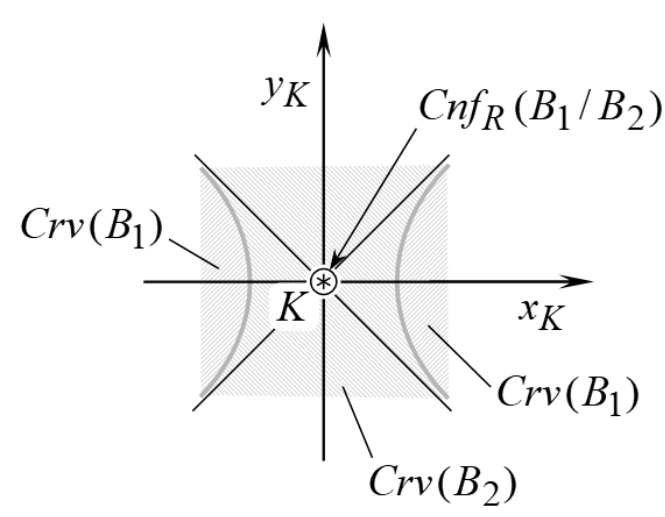

Minimal-to-minimal (pseudo-umbilic)

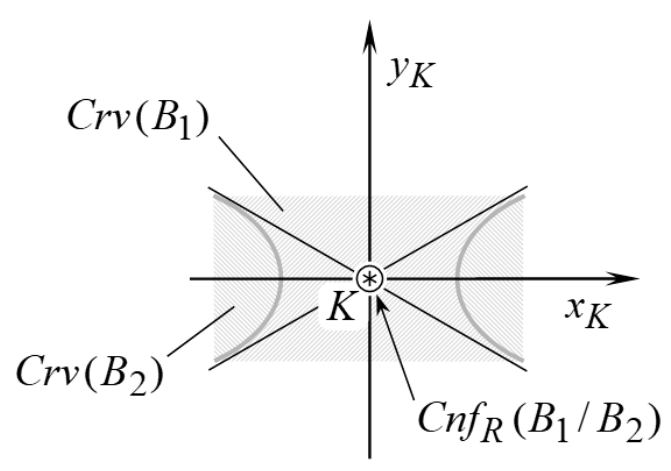

Hyperbolic (pseudo-convex)-tohyperbolic (pseudo-concave)

\section{9}

10.8

Fig. 29. Indicatrices of conformity, $\operatorname{Cnf}_{R}\left(B_{1} / B_{2}\right)$, and the curvature indicatrices, $\operatorname{Crv}\left(B_{1}\right)$ and $\operatorname{Crv}\left(B_{2}\right)$, at high-conformal point-contact kinematic pairs II kinematic pairs.

At one of the principal sections, the functional surfaces make line contact, while in the other principal plane section an inequality, $d_{c n f} \leq\left[d_{c n f}^{\min }\right]$, is observed [in all the contacts, the actual value of the angle, $\mu$, of the functional surfaces, $B_{1}$ and $B_{2}$, local relative orientation, equals $\mu= \pm 180^{\circ}$ ). In Fig. 7, kinematic pairs of this kind correspond to 3.1, 7.5, 9.9, and 10.8, contacts of the functional surfaces $B_{1}$ and $B_{2}$. Relative orientation of the curvature indicatrices, $\operatorname{Crv}\left(B_{1}\right)$ and $\operatorname{Crv}\left(B_{2}\right)$, at contact point of the kinematic pairs of this particular kind are shown in Fig. 29. For the kinematic pairs under consideration, the actual value of the angle, $\mu$, of the functional surfaces, $B_{1}$ and $B_{2}$, local relative orientation equals to a value $\mu= \pm 180^{\circ}$.

The indicatrices of conformity, $\operatorname{Cnf}_{R}\left(B_{1} / B_{2}\right)$, for kinematic pairs featuring locally-surface-tosurface_II-contact of the functional surfaces, $B_{1}$ and $B_{2}$, is always shrunk to a point. This point is coincident with the contact point of the functional surfaces.

6.3. Surface-to-surface-contact kinematic pairs. In surface-to-surface-contact kinematic pairs (or just $S S_{c}$-kinematic pairs, for simplicity), functional surfaces, $B_{1}$ and $B_{2}$, make contact within a common surface patch ${ }^{5}$. A more in detail analysis reveals that following kinds of contact between the functional surfaces, $B_{1}$ and $B_{2}$, have to recognized in surface-to-contact kinematic pairs.

Considered locally, surface-to-surface-contact kinematic pairs cannot be distinguished from the shown in Fig. 17, locally-surface-to-surface_I-contact kinematic pairs, and from the shown in Fig. 28 locally-surface-to-surface_II-contact kinematic pairs. Indicatrix of conformity at any point of contact of the functional surfaces in surface-to-surface-contact kinematic pairs is shrunk to a point. Moreover, true-surface-to-surface-contact kinematic pairs can be viewed as a reduced case of any of the shown in see Fig. 7. Kinematic pairs 1.1, 2.1, 2.2, 3.1, .., 10.9, and 10.10.

\footnotetext{
${ }^{5}$ Kinematic pairs of the design under consideration, can also be referred to as true surface-to-surface contact kinematic pairs (or just TSS T $^{-}$ kinematic pairs, for simplicity). 
To avoid indefinites, the following is helpful to be taken into account.

In a kinematic pair, the functional surfaces, $B_{1}$ and $B_{2}$, have to have at least one degree of freedom in relation to each other. Therefore, surfaces not of all geometries can be used in the design of kinematic pairs of the kind under consideration. In order to be suitable for application in the design of kinematic pairs that feature surface-to-surface-contact of the functional surfaces, a functional surface has to allow sliding over itself.

The property of surface $B$ to allow for sliding over itself means that for the surface $B$ there exists a corresponding motion: when performing this motion, the enveloping to the consecutive positions of the traveling surface $B$ is congruent to the surface $B$ itself.

According to that, the following functional surfaces that allow for sliding over themselves, are recognized:

- A screw surface of a constant axial pitch $\left(p_{x}=\right.$ Const $)$ - one degree of freedom

- A surface of revolution about the centerline of the surface of revolution (the axial pitch of a screw surface is reduced to a zero $p_{x}=0$ ) - one degree of freedom

- A surface of translation along the directrix (the axial pitch of a screw surface approaches to an infinity $\left.p_{x} \rightarrow \infty\right)$ - one degree of freedom

In addition to that, a few reduced cases of the surfaces that allow for sliding over themselves, can also be distinguished:

- A cylinder of revolution (a cylinder of revolution allows a rotation about, as well as a straight motion along the centerline of the cylinder) - two degrees of freedom

- A sphere (a sphere allows for three rotations about three axes independently) - three degrees of freedom

- A plane (a plane surface allows straight motion in two different directions within the plane, as well as a rotation about an axis that is perpendicular to the plane) - three degrees of freedom

It is proven [2], [3], [4], [7] that there are no other kinds of functional surfaces that allow for sliding over themselves.

From the standpoint of surface geometry, kinematic pairs those composed of two screw surfaces are more complex (and are more general) compared to kinematic pairs composed either of the sphereto-sphere surfaces (having a single degree of freedom), or of the plane-to-plane surfaces (both having three degrees of freedom).

A performed analysis of possible kinds of kinematic pairs composed of two surfaces congruent to one another, reveals that there are only 6 possible kinds of kinematic pairs of this particular design. The kinematic pairs of all of these designs form a separate group of kinematic pairs.

There are six different kinds of kinematic pairs composed by two congruent local patches of functional surfaces, $B_{1}$ and $B_{2}$ :

- Screw-to-screw-contact kinematic pair (one DoF)

- Surface-of-rotation-to-surface-of-rotation-contact kinematic pair (one DoF)

- Surface-of-translation-to-surface-of-translation-contact kinematic pair (one DoF)

- Cylinder-of-rotation-to-cylinder-of-rotation-contact kinematic pair (two DoF)

- Sphere-to-sphere-contact kinematic pair (three DoF)

- Plane-to-plane-contact kinematic pair (three DoF)

True-surface-to-surface-contact kinematic pairs of no other design are feasible.

\section{Classification of Kinematic Pairs}

Depending of the kind of contact of two functional surfaces, the following groups of kinematic pairs are recognized:

$P_{c}$ - kinematic pairs (or point-contact kinematic pairs): 


\section{Bulletin of Science and Technical Development}

www.vntr.ru

№ 161, 2021

$T P_{c}$-kinematic pairs (or true-point-contact kinematic pairs) - 29 kinds in total;

$L L_{c}$ - kinematic pairs (or locally-line-contact kinematic pairs) - 20 kinds in total;

$L S_{c .1}$-kinematic pairs (or locally-surface-to-surface_I-contact kinematic pairs) - 7 kinds in total;

$H C_{c .1}$ - kinematic pairs (or high-conformal-contact_I kinematic pairs) - 20 kinds in total; $L_{c}$-kinematic pairs (or line-contact kinematic pairs):

$T L_{c}$ - kinematic pairs (or true-line-contact kinematic pairs) - 23 kinds in total;

$L S_{c .2}$ - kinematic pairs (or locally-surface-to-surface_II-contact kinematic pairs) - 8 kinds in total;

$H C_{c .2}$ - kinematic pairs (or high-conformal-contact_II kinematic pairs) - 8 kinds in total; $S S_{c}$ - kinematic pairs (or surface-to-surface-contact kinematic pairs):

$T_{S S}$ - kinematic pairs (or true-surface-to-surface-contact kinematic pairs) - 6 kinds in total; The following classification of the discussed kinds of kinematic pairs, both, all earlier known, as well as all newly introduced kinematic pairs, are covered by the proposed classification.

Only $29+23+6+20+7+8+20+7+8=128$ kinds $^{6}$ of contact of two smooth, regular surfaces $B_{1}$ and $B_{2}$ are possible in nature ${ }^{7}$. For some kinds of the surfaces contact, no constraints are imposed on the actual value of the angle $\mu$ of local relative orientation of the functional surfaces, $B_{1}$ and $B_{2}$. For the rest kinds of the functional surfaces contact, a corresponding range of the allowed value of the angle $\mu:\left[\mu_{\min }\right] \leq \mu \leq\left[\mu_{\max }\right]$ is specified. For particular cases of the functional surfaces contact, the only feasible value $\mu=[\mu]$ is allowed.

\footnotetext{
${ }^{6}$ It is desirable to have these numbers verified by an independent researcher(s).

${ }^{7}$ The results of a more in detail investigation of all possible kinds of true point contact of two smooth, regular surfaces in the first order of tangency can be found in [13], [15], and [19]. 


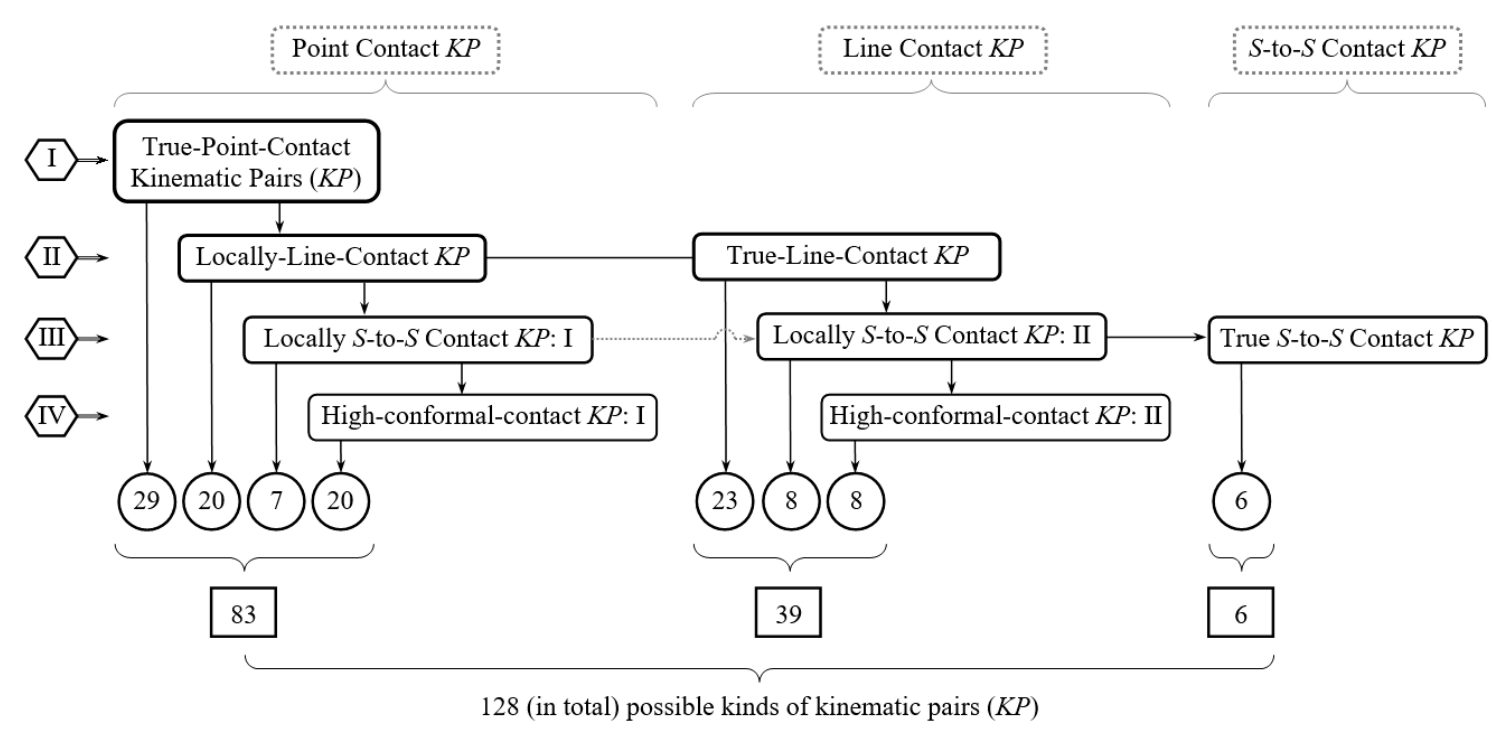

Fig. 30. Classification of possible kinds of kinematic pairs: There are as many as 128 (in total) different kinds of kinematic pairs $(K P) ; S$ - to $-S$ stands for Surface-to-Surface contact of the functional surfaces.

The proposed classification of possible kinds of kinematic pairs is illustrated by a chart shown in Fig. 30. As seen from Fig. 30, four stratums (I through IV) are distinguished in the classification. There are as many as 128 (in total) different kinds of kinematic pairs. The proposed classification (see Fig. 30) is self-consistent and complete (or, at least, potentially complete).

A more in-detail analysis of the kinematic pairs of all the geometries listed in Fig. 30 is desirable. In reality, the functional surfaces, $B_{1}$ and $B_{2}$, in a kinematic pair are displaced in relation to one another (which is inevitable). The total displacement of the functional surfaces can be expressed in terms of (a) three elementary linear displacements $\left(\delta_{x}, \delta_{y}, \delta_{z}\right)$ along the axes of a local reference system, $x_{K} y_{K} z_{K}$, with the origin at contact point, $K$, and (b) three elementary angular displacements $\left(\varphi_{x}, \varphi_{y}, \varphi_{z}\right)$ about the axes of the local reference system, $x_{K} y_{K} z_{K}$. If the linear, $\delta_{x}$, $\delta_{y}$, and $\delta_{z}$, and the angular, $\varphi_{x}, \varphi_{y}$, and $\varphi_{z}$, displacements are taken into account, the so-called quasi-surface-to-surface-contact kinematic pairs of the first (I), and of the second (II) kind can be introduced into consideration. Quasi-surface-to-surface-contact kinematic pairs of the first (I) kind feature single contact point, $K$. Quasi-surface-to-surface-contact kinematic pairs of the second (II) kind feature a common contact line, LC. Incorporation of the quasi-surface-to-surface-contact kinematic pairs of the first (I), and of the second (II) kind into the analysis represents an additional way for further evolution of the proposed classification of the kinematic pairs (see Fig. 30). This potential concept is not covered in this text, and it may be a subject for future work.

\section{Concluding Remarks}

This paper deals with kinematic pairs. Possible kinds of kinematic pairs are investigated. Novel kinds of kinematic pairs are discovered. Correspondence between normal curvatures of functional surfaces in kinematic pairs is investigated. Instead of two groups of kinematic pairs commonly recognized in the nowadays practice (that is, instead of the so-called lower kinematic pairs, and higher kinematic pairs), it is proposed to distinguish three groups of kinematic pairs, namely:

(a) point-contact kinematic pairs (or just $P_{c}$-kinematic pairs, for simplicity)

(b) line-contact kinematic pairs (or just $L_{c}$-kinematic pairs, for simplicity)

(c) surface-to-surface-contact kinematic pairs (or just $S_{c}$-kinematic pairs, for simplicity) 
www.vntr.ru

№ 161, 2021

The concept of three groups of kinematic pairs is originated from two patents [11] (of 1983) and [12] (of 1984), and is based on the research undertaken by the author in the late 1970s.

A novel classification of all possible kinds of kinematic pairs is proposed. The proposed classification is potentially complete, and is open for further improvements.

Proper labeling can be used for identification of kinematic pairs: instead of the indefinite terms higher kinematic pair and lower kinematic pair, appropriate labels (for instance, the kinematic pair \#10.5) can used instead.

A more in-detail analysis of kinematic pairs of all of the listed geometries is possible now.

\section{Funding of Research}

This research did not receive any specific grant from funding agencies in the public, commercial, or not-for-profit sectors.

\section{References}

[1] Cauchy, A.L., Leçons sur les Applications du Calcul Infinitésimal á la Geometrie, Paris, Imprimerie royale, 1826.

[2] Darboux, G., Sur le Déplacement d'une Figure Invariable, Comptes rendus de l'Académie des science, XCII, 1981, pp.118-121. [Quoted after: Bottema, O. and Roth, B., Theoretical Kinematics, Dover Publications, Inc., New York, 1990, 558 pages (page 304)].

[3] Denavit, J. and Hartenberg, R.S., "A Kinematics Notation for Lower-Pair Mechanisms Based on Matrices", ASME Journal of Applied Mechanics, Vol. 77, 1955, pp. 215-221.

[4] doCarmo, M.P., Differential Geometry of Curves and Surfaces, Prentice-Hall, Englewood Cliffs, NJ, 1976, 503p.

[5] Dvornikov, L.T., "Fundamentals of the theory of geometric connections (kinematic pairs) in mechanical systems", Theory of Mechanisms and Machines, 2014, Vol. 12, No. 2, pp. 25-49. http://tmm.spbstu.ru/24/3Dvornikov.pdf

[6] Dvornikov, L.T., Theory of Kinematic Pairs, Novokuznetsk, Siberia State Industrial University Publishers, 2001.

[7] Eisenhart, L.P., A Treatise on the Differential Geometry of Curves and Surfaces, Dover Publications, Inc., London, 1909; New York, reprint 1960, 474p.

[8] Favard, J., Course de Gèomètrie Diffèrentialle Locale, Gauthier-Villars, Paris, 1957, viii $+553 \mathrm{p}$.

[9] Hartenberg, R.S. and Denavit, J., Kinematic synthesis of linkages, New York, McGraw-Hill, 1964, pp. 17-18.

[10] Lagrange, J.L., Theorié des Fonctions Analytiques, Paris, Impr. De la République, prairial an $\mathrm{V}, 1797,277 \mathrm{p}$.

[11] Pat. No. 1,185,749, USSR, A Method of Sculptured Surface Machining on a Multi-Axis NC Machine./S.P. Radzevich, Int. Cl. B23c 3/16, Filed: October 24, 1983.

[12] Pat. No. 1,249,787, USSR, A Method of Sculptured Surface Machining on a Multi-Axis NC Machine./S.P. Radzevich, Int. Cl. B23c 3/16, Filed: December 27, 1984.

[13] Radzevich, S.P., Generation of Surfaces: Kinematic Geometry of Surface Machining, CRC Press, Boca Raton, Florida, 2014, 738 pages.

[14] Radzevich, S.P., Geometry of Surfaces: A Practical Guide for Mechanical Engineers, $2^{\text {nd }}$ edition, Springer International Publishing, 2019, (C) Springer Nature Switzerland AG (2020), XXVI, 329 pages, . (First edition: Wiley, 2013, 264 pages).

[15] Radzevich, S.P., High-Conformal Gearing: Kinematics and Geometry, $2^{\text {nd }}$ edition, Elsevier, Amsterdam, 2020, 506 pages. [ $1^{\text {st }}$ edition: $C R C$ Press, Boca Raton, Florida, 2015, 332 pages]. 
[16] Radzevich, S.P., "Kinematic Pairs: Novel Kinds and Classification", pages 89-142 in: Radzevich, S.P., editor, Recent Advances in Gearing: Scientific Theory and Applications, Springer International Publishing, 2021, 566 pages.

[17] Radzevich, S.P., "Mathematical Modeling of Contact of Two Surfaces in the First Order of Tangency", Mathematical and Computer Modeling; Vol.39, Issue 9-10, May 2004, pp.10831112.

[18] Radzevich, S.P., Methods of Analysis of Contact Geometry of Free-Form-Part Surface and the Generating Surfaces of the Cutting Tool, Dneprodzerzhinsk, DII (UkrNIINTI, 30.03.1988, No. 759-Uk88), 1987, 103 pages.

[19] Radzevich, S.P., Theory of Gearing: Kinematics, Geometry, and Synthesis, $2^{\text {nd }}$ Edition, revised and expanded, CRC Press, Boca Raton, FL, 2018, 934 pages. (First edition: Radzevich, S.P., Theory of Gearing: Kinematics, Geometry, and Synthesis, CRC Press, Boca Raton, Florida, 2012, 743 pages).

[20] Reuleaux, F., Lehrbuch der Kinematik, Braunschweig, 1875, Bd. [Reuleaux, F., The kinematics of machinery, 1876, (translated and annotated by A.B.W. Kennedy), reprinted by Dover, New York, 1963].

[21] Sacs, E., Joskowicz, L., "Computational Kinematic Analysis of Higher Pairs with Multiple Contacts", Journal of Mechanism Design, 1995, Vol. 117, pages 269-277.

[22] Snidle, H.P., Evans, H.P., "Elastohydrodynamic Lubrication of Real Surfaces", Tribology Series, Vol. 30, 1995, pp. 219-227.

[23] Usher, A.P., A History of Mechanical Inventions, Harvard University Press, 1929, (reprinted by Dover Publications, 1968).

[24] Zhivago, E.Ya., Theory and Systematization of Kinematic Pairs of Mechanical Systems, Dr.Sci Thesis, Siberia State Industrial University, Novokuznetsk, 2000, 261 pages.

\section{Bibliography}

Dvornikov, L.T., Zhivago, E.Ya., Fundamentals of the Theory of Kinematic Pairs: The Monograph, Novokuznetsk, SbGIU Publishers, 1999, 105 pages.

Ertel, A.M, Hydrodynamic Calculation of Lubrication of Spatial Surfaces Contact (Gear Meshes, Rolling Bearings, Extremely Heavily Loaded Journals, and so forth), Moscow, TsNIITMash, 1945, No. 1, 64 pages.

Ertel, A.M, "Hydrodynamic Theory of Lubrication in New Applications", Applied Mathematics and Mechanics, 1939, Vol. 3, No. 2, pp. 41-49.

Khruschov, M.M., "On the History of the Development of the Theory of Elastohydrodynamic Lubrication", Friction and Wear, 1996, Vol. 17, No. 5, pp. 703-706.

Krishna, R.K., Sen, D., "Second-order total freedom analysis of 3D objects in a single point contact", Mechanism and Machine Theory, Vol. 140, October 2019, pages 10-30.

Mohrenstein-Ertel, A., "Die Berechnung der hydrodynamischen Schmierung gekrümmter Oberflüchen unter hoher Belastung und Relativbewegung”, Fortschr.-Ber. VDI-Z, Reihe 1, Nr. 115, Düsseldorf, 1984, s. 85-89.

Radzevich, S.P., “A Possibility of Application of Plücker's Conoid for Mathematical Modeling of Contact of Two Smooth Regular Surfaces in the First Order of Tangency", Mathematical and Computer Modeling, Vol. 42, Issues 9-10, November 2005, pp.999-1022.

Radzevich, S.P., Fundamentals of Surface Generation, Monograph, Kiev, Rastan, 2001, 592p. (In Russian).

Radzevich, S.P., Kinematic Geometry of Surface Machining, CRC Press, Boca Raton, FL 2007, 508p. 
Radzevich, S.P., "On Analytical Description of the Geometry of Contact of Surfaces in Higher Kinematic Pairs", Theory for Mechanisms and Machines, 2005, No. 2, Vol. 3, pp.3-14. http://tmm.spbstu.ru

Zhivago, E.Ya., Fundamentals of the Theory of Kinematic Pairs, Sci.Dr. Thesis, 05.02.18: Theory of Mechanisms and Machine, SibSIU, Novokuznetsk, 2000, 261 pages.

Дата поступления: 6 апреля 2021 г. 